\title{
ENLACEMENTS DU MOUVEMENT BROWNIEN AUTOUR DES COURBES DE L'ESPACE
}

\author{
JEAN-FRANCOIS LE GALL ET MARC YOR
}

\begin{abstract}
Limit theorems are proved for the winding numbers of a threedimensional Brownian motion around certain curves in space. In particular, the joint asymptotic distribution of the winding numbers around two curves is obtained. This joint distribution generalizes the asymptotic law of the winding numbers of a planar Brownian motion around two points, which has recently been given by Pitman and Yor. The limiting distributions are closely related to the time spent by a linear Brownian motion above and below a multiple of its maximum process. Proofs rely on stochastic calculus for continuous semimartingales.
\end{abstract}

\section{INTRODUCTION}

Soit $B=(X, Y, Z)$ un mouvement brownien à valeurs dans $\mathbf{R}^{3}$. Le but principal du présent travail est l'étude asymptotique du nombre de tours de $B$ autour d'une courbe $(\Gamma)$ de l'espace. Nous nous restreindrons à une classe assez particulière de courbes: nous supposerons que la courbe $(\Gamma)$ est donnée par une équation de la forme $(x, y)=f(z)$, où la fonction $f: \mathbf{R} \rightarrow \mathbf{R}^{2}$ est de classe $C^{2}$ et vérifie certaines hypothèses précisées plus loin.

Soit $W$ le processus plan $W_{t}=\left(X_{t}, Y_{t}\right)$. Nous appellerons processus d'enlacement de $B$ autour de la courbe $(\Gamma)$ toute détermination continue $\theta=\left(\theta_{t}, t \geq 0\right)$ de l'argument de $W_{t}-f\left(Z_{t}\right)$. Le choix de la valeur initiale $\theta_{0}$ importe peu puisque nous nous intéressons à des propriétés asymptotiques. Nous noterons simplement:

$$
\theta_{t}=\operatorname{Arg}\left(W_{t}-f\left(Z_{t}\right)\right)
$$

Pour que cette définition ait un sens, il est nécessaire que $W_{0} \neq f\left(Z_{0}\right)$ p.s. Inversement, si cette propriété est satisfaite, on a $W_{t} \neq f\left(Z_{t}\right)$ pour tout $t$, p.s. En effet, la courbe $(\Gamma)$ est de capacité newtonienne nulle, puisqu'elle est réunion dénombrable d'ensembles de mesure de Hausdorff linéaire finie.

Notre définition du processus d'enlacement est facile à justifier dans le cas particulier où $(\Gamma)$ est une courbe plane. Supposons par exemple $f(z)=$

Received by the editors February 24, 1988 and, in revised form, June 14, 1988.

1980 Mathematics Subject Classification (1985 Revision). Primary 60J55; Secondary 60F05, $60 \mathrm{H} 05$.

Key words and phrases. Asymptotic distributions, Brownian motion, excursions, stochastic integrals, winding numbers. 
$\left(f_{1}(z), 0\right)$, pour tout $z \in \mathbf{R}$. La courbe $(\Gamma)$ est contenue dans le plan $\{y=0\}$ et délimite dans ce plan les deux régions:

$$
P_{1}=\left\{(x, 0, z) ; x<f_{1}(z)\right\}, \quad P_{2}=\left\{(x, 0, z) ; x>f_{1}(z)\right\} .
$$

Définissons par récurrence

$$
T_{0}=0, \quad T_{2 i+1}=\inf \left\{t>T_{2 i} ; B_{t} \in P_{1}\right\}, \quad T_{2 i+2}=\inf \left\{t>T_{2 i+1} ; B_{t} \in P_{2}\right\},
$$

et posons

$$
\begin{aligned}
\varepsilon_{2 i} & =\left\{\begin{array} { r } 
{ + 1 } \\
{ - 1 }
\end{array} \text { selon que } Y _ { s } \left\{\begin{array}{l}
<0 \\
>0
\end{array} \text { quand } s \rightarrow T_{2 i+1}, s<T_{2 i+1},\right.\right. \\
\varepsilon_{2 i+1} & =\left\{\begin{array} { r } 
{ + 1 } \\
{ - 1 }
\end{array} \text { selon que } Y _ { s } \left\{\begin{array}{l}
>0 \\
<0
\end{array} \text { quand } s \rightarrow T_{2 i+2}, s<T_{2 i+2} .\right.\right.
\end{aligned}
$$

La quantité $N_{t}=\sum_{\left\{i ; T_{i} \leq t\right\}} \varepsilon_{i}$ représente le nombre de demi-tours (comptés avec leur signe) effectués par $B$ autour de $(\Gamma)$ avant l'instant $t$. On voit aisément que $\left|\theta_{t}-\pi N_{t}\right| \leq$ const., ce qui montre que l'étude asymptotique de $N_{t}$ équivaut à celle de $\theta_{t}$.

Lorsque $f$ est une fonction constante, l'étude asymptotique de $\theta_{t}$ est celle du nombre de tours d'un mouvement brownien plan autour d'un point. Ce problème a été étudié par de nombreux auteurs: voir notamment Spitzer [19], Williams [20], Durrett [2, 3], et Messulam-Yor [16]. D'après [19], on a dans ce cas particulier,

$$
\frac{2}{\log t} \theta_{t} \underset{t \rightarrow \infty}{\stackrel{(d)}{\rightarrow}} C_{1}
$$

où $C_{1}$ désigne une variable de Cauchy de paramètre 1 et $(d)$ indique la convergence en distribution. Notre premier objectif est d'étendre le résultat limite (1.a) à la situation plus générale de l'enlacement de $B$ autour d'une courbe $(x, y)=f(z)$. Nous montrons (Théorème 4.2) que (1.a) reste vrai à condition que $f$ satisfasse l'hypothèse suivante:

$$
\left\{\begin{array}{l}
\text { (i) Il existe deux constantes } C>0 \text { et } \alpha>0 \text { telles que, pour } \\
\text { tout } z \in \mathbf{R},\left|f^{\prime}(z)\right| \leq C|z|^{-\alpha}, \\
\text { (ii) } \int_{-\infty}^{+\infty}\left|f^{\prime \prime}(z)\right| d z<\infty .
\end{array}\right.
$$

L'assertion (i) entraîne, éventuellement avec un autre choix de la constante $C$ que, pour tout $z \in \mathbf{R}$,

$$
|f(z)| \leq C\left(1+|z|^{\beta}\right)
$$

en posant $\beta=1-\alpha$, si $\alpha \neq 1$, et en choisissant $\beta>0$ de façon arbitraire, si $\alpha=1$. La courbe $(\Gamma)$ admet donc pour direction asymptotique la droite $(D)$ définie par l'équation $x=y=0$. Il est alors naturel de chercher à comparer les enlacements de $B$ autour de $(\Gamma)$ et $(D)$. A nouveau, le cas très particulier de ce problème où $(\Gamma)$ est simplement une droite parallèle à $(D)$ a déjà été étudié: on est alors ramené à l'étude asymptotique des nombres de tours d'un mouvement brownien plan autour de deux points. Ce problème a été traité 
en détail par Pitman-Yor [18] (voir aussi Le Gall-Yor [12]). En revenant à la situation générale, on peut se demander, dans le cas où $(\Gamma)$ admet $(D)$ pour asymptote (i.e., $\lim _{|z| \rightarrow \infty}|f(z)|=0$ ), si les enlacements de $B$ autour de $(\Gamma)$ et $(D)$ sont "asymptotiquement proches." Précisément, si $\theta^{0}$ désigne l'enlacement de $B$ autour de $(D)$, a-t-on

$$
\frac{1}{\log t}\left(\theta_{t}-\theta_{t}^{0}\right) \underset{t \rightarrow \infty}{\stackrel{(P)}{\rightarrow}} 0 ?
$$

Une réponse assez satisfaisante à cette question est donnée dans la partie 5 , où l'on étudie plus généralement la loi jointe des enlacements, notés $\theta, \theta^{\prime}$, de $B$ autour de deux courbes $(\Gamma),\left(\Gamma^{\prime}\right)$ associées respectivement à des fonctions $f$ et $g$ qui satisfont l'hypothèse $(\mathrm{H})$. Sous l'hypothèse supplémentaire que

$$
\lim _{|z| \rightarrow \infty} \frac{1}{\log |z|} \log |f(z)-g(z)|=a \in[-\infty ; 1[
$$

nous montrons (Théorème 5.1) que

$$
\frac{2}{\log t}\left(\theta_{t}, \theta_{t}^{\prime}\right) \underset{t \rightarrow \infty}{\stackrel{(d)}{\rightarrow}}\left(\omega_{1}\left(\sigma_{1}\right)+\omega_{2}\left(\sigma_{2}\right), \omega_{1}^{\prime}\left(\sigma_{1}^{\prime}\right)+\omega_{2}\left(\sigma_{2}\right)\right)
$$

où $\omega_{1}, \omega_{1}^{\prime}, \omega_{2}$ sont trois mouvements browniens réels issus de 0 , indépendants et indépendants de $\sigma_{1}, \sigma_{1}^{\prime}, \sigma_{2}$, et la loi du triplet $\left(\sigma_{1}, \sigma_{1}^{\prime}, \sigma_{2}\right)$ (qui dépend de $a)$ peut être décrite comme suit. Soient $\beta=\left(\beta_{t}, t \geq 0\right)$ un mouvement brownien réel issu de 0 et $\hat{\beta}$ le processus du maximum de $\beta: \hat{\beta}_{t}=$ $\sup \left\{\beta_{s}, s \leq t\right\}$. Soient aussi $\sigma=\inf \left\{t \geq 0 ; \beta_{t}=1\right\}$ et $l_{\sigma}^{(a)}$ le temps local en 0 , à l'instant $\sigma$, de la semi-martingale $\beta-a \hat{\beta}$. Le triplet $\left(\sigma_{1}, \sigma_{1}^{\prime}, \sigma_{2}\right)$ a même loi que

$$
\left(\frac{1}{4}\left(l_{\sigma}^{(a)}\right)^{2} T_{1}, \frac{1}{4}\left(l_{\sigma}^{(a)}\right)^{2} T_{2}, \int_{0}^{\sigma} I\left(\beta_{s} \geq a \hat{\beta}_{s}\right) d s\right),
$$

où $T_{1}, T_{2}$ désignent deux variables aléatoires indépendantes et indépendantes du mouvement brownien $\beta$, et ayant même loi que $\sigma$, c'est-à-dire

$$
P(U \in d t)=\frac{1}{\left(2 \pi t^{3}\right)^{1 / 2}} e^{-1 / 2 t} d t, \quad \text { pour } U=T_{1}, T_{2} \text { ou } \sigma .
$$

En particulier, chacun des deux couples $\left(\sigma_{1}, \sigma_{2}\right),\left(\sigma_{1}^{\prime}, \sigma_{2}\right)$ a même loi que

$$
\left(\int_{0}^{\sigma} I\left(\beta_{s}<a \hat{\beta}_{s}\right) d s, \int_{0}^{\sigma} I\left(\beta_{s} \geq a \hat{\beta}_{s}\right) d s\right) .
$$

La loi limite de (1.d) peut aussi être caractérisée par sa transformée de Fourier $E\left[\exp i\left(\lambda_{1} \omega_{1}\left(\sigma_{1}\right)+\lambda_{1}^{\prime} \omega_{1}^{\prime}\left(\sigma_{1}^{\prime}\right)+\lambda_{2} \omega_{2}\left(\sigma_{2}\right)\right)\right]=\left(\operatorname{ch} \lambda_{2} \bar{a}+\left(\left|\lambda_{1}\right|+\left|\lambda_{1}^{\prime}\right|\right) \frac{\operatorname{sh} \lambda_{2} \bar{a}}{\lambda_{2}}\right)^{-1 / \bar{a}}$, où $\bar{a}=1-a$, et nous utilisons ici la notation française: $\operatorname{ch} x=\frac{1}{2}\left(e^{x}+e^{-x}\right)$, $\operatorname{sh} x=\frac{1}{2}\left(e^{x}-e^{-x}\right)$. L'étude de cette loi limite conduit à de nombreuses questions intéressantes sur le mouvement brownien linéaire et son processus du 
maximum. Ces problèmes sont abordés dans la partie 6 et seront développés dans un article à venir [14].

Les deux variables limites qui interviennent dans (1.d) coïncident seulement lorsque $\sigma_{1}=\sigma_{1}^{\prime}=0$, ce qui se produit si et seulement si $a=-\infty$. Autrement dit, sous l'hypothèse (1.c), la famille $(\log t)^{-1}\left(\theta_{t}-\theta_{t}^{\prime}\right)$ converge en probabilité vers 0 si et seulement si $a=-\infty$. En revenant au cas particulier où $\left(\Gamma^{\prime}\right)$ est la droite $(D)$, on voit qu'une condition suffisante pour que la convergence (1.b) soit réalisée est

$$
\text { pour tout } a>0, \quad \lim _{|z| \rightarrow \infty}|z|^{a}|f(z)|=0 .
$$

Lorsque $a>-\infty$, le résultat (1.d) met en évidence une décomposition asymptotique de l'enlacement $\theta_{t}$ qui est très semblable à la décomposition en "grand angle" et "petit angle" introduite par Messulam et Yor [16] et utilisée systématiquement par Pitman et Yor [18] dans l'étude des nombres de tours du mouvement brownien plan. Le rôle du grand angle est ici tenu par la variable $\omega_{2}\left(\sigma_{2}\right)$ qui représente les enlacements communs de $B$ autour des deux courbes $(\Gamma)$ et $\left(\Gamma^{\prime}\right)$ (un peu comme le grand angle prend en compte les tours effectués par un mouvement brownien plan simultanément autour de deux, ou plusieurs, points). Le rôle du petit angle est joué par la variable $\omega_{1}\left(\sigma_{1}\right)$ qui représente les "enlacements spécifiques" de $B$ autour de $(\Gamma)$. Insistons sur le fait que l'expression "enlacements spécifiques" n'a de sens que parce qu'on s'est donné une seconde courbe $\left(\Gamma^{\prime}\right)$ et qu'on distingue entre les enlacements communs à $(\Gamma)$ et $\left(\Gamma^{\prime}\right)$ et les autres.

Revenons à la situation où $g \equiv 0$, i.e. $\left(\Gamma^{\prime}\right)$ est la droite $(D)=\{x=y=0\}$. La condition (1.b) est certainement satisfaite si on impose la condition plus forte

$$
\left(\theta_{t}-\theta_{t}^{0}, t \rightarrow \infty\right) \text { converge p.s. }
$$

qui a lieu si et seulement si

$$
\lim _{t \rightarrow \infty} \frac{\left|f\left(Z_{t}\right)\right|}{\left|W_{t}\right|}=0, \quad \text { p.s. }
$$

Supposons pour simplifier que, au moins pour $z$ assez grand, on ait $|f(z)|=$ $|z|^{1 / 2} h(|z|)$, où $h:[0 ; \infty[\rightarrow[0 ; \infty[$ est décroissante. Une application simple du Théorème 1 de Spitzer [19] montre que la convergence (1.e) a lieu si et seulement si

$$
\int^{\infty} \frac{d u}{u|\log h(u)|}<\infty
$$

On voit facilement que la condition (1.c) écrite avec $g \equiv 0$ et $a=-\infty$ est satisfaite par de nombreuses fonctions $f$ qui ne vérifient pas (1.g). En d'autres mots, les angles autour de $(\Gamma)$ et $(D)$ peuvent être asymptotiquement identiques, au sens où (1.b) est vérifiée, sans que la convergence (1.e) ait lieu. 
Une question très naturelle, qui nous a été posée par le referee, consiste à remplacer la condition (1.c) par

$$
\left\{\begin{array}{l}
\lim _{z \rightarrow+\infty} \frac{1}{\log z} \log |f(z)-g(z)|=a_{1}, \\
\lim _{z \rightarrow-\infty} \frac{1}{\log |z|} \log |f(z)-g(z)|=a_{2},
\end{array}\right.
$$

où $a_{1}, a_{2} \in[-\infty ; 1[$ (et ne sont pas en général égaux). Les arguments de la partie 5 suggèrent que, sous l'hypothèse ( $\left.1 . c^{\prime}\right)$, la convergence (1.d) a encore lieu, la loi limite étant maintenant caractérisée par:

$$
\begin{aligned}
& E\left[\exp i\left(\lambda_{1} \omega_{1}\left(\sigma_{1}\right)+\lambda_{1}^{\prime} \omega_{1}^{\prime}\left(\sigma_{1}^{\prime}\right)+\lambda_{2} \omega_{2}\left(\sigma_{2}\right)\right)\right] \\
& \quad=\left(\operatorname{ch} \lambda_{2} \bar{a}_{1}+\left(\left|\lambda_{1}\right|+\left|\lambda_{1}^{\prime}\right|\right) \frac{\operatorname{sh} \lambda_{2} \bar{a}_{1}}{\lambda_{2}}\right)^{-1 / 2 \bar{a}_{1}}\left(\operatorname{ch} \lambda_{2} \bar{a}_{2}+\left(\left|\lambda_{1}\right|+\left|\lambda_{1}^{\prime}\right|\right) \frac{\operatorname{sh} \lambda_{2} \bar{a}_{2}}{\lambda_{2}}\right)^{-1 / 2 \bar{a}_{2}}
\end{aligned}
$$

où $\bar{a}_{1}=1-a_{1}, \bar{a}_{2}=1-a_{2}$. Nous ne développerons pas ici cette généralisation, qui paraît nécessiter un travail technique sensiblement plus important que celui de la preuve de (1.d).

Les preuves de (1.a) et (1.d) reposent essentiellement sur le calcul stochastique. Si $\left(\Phi_{t} ; t \geq 0\right)$ désigne une détermination continue du logarithme complexe le long de la courbe $\left(W_{t}-f\left(Z_{t}\right) ; t \geq 0\right)$, c'est-à-dire

$$
\Phi_{t}=\log \left|W_{t}-f\left(Z_{t}\right)\right|+i \theta_{t},
$$

on a

$$
\Phi_{t}-\Phi_{0}=\int_{\left(W_{u}-f\left(Z_{u}\right) ; u \leq t\right)} d \xi / \xi \underset{(*)}{=} \oint_{0}^{t} \frac{d\left(W_{u}-f\left(Z_{u}\right)\right)}{W_{u}-f\left(Z_{u}\right)},
$$

où le signe $\oint$ désigne l'intégrale stochastique au sens de Stratonovitch (l'intégrale de $d \xi / \xi$ le long de la courbe $\left(W_{u}-f\left(Z_{u}\right), u \leq t\right)$ est la limite en probabilité des intégrales le long d'une suite d'approximations polygonales, et cette limite est une intégrale de Stratonovitch; pour une preuve détaillée de l'identité $(*)$, voir par exemple Yor [22]). On a donc

$$
\Phi_{t}-\Phi_{0}=\int_{0}^{t} \frac{d\left(W_{u}-f\left(Z_{u}\right)\right)}{W_{u}-f\left(Z_{u}\right)}-\frac{1}{2} \int_{0}^{t}\left(\frac{f^{\prime}\left(Z_{u}\right)}{W_{u}-f\left(Z_{u}\right)}\right)^{2} d u,
$$

la première intégrale étant maintenant une intégrale d'Itô. Ensuite, une observation clé est que, dans le membre de droite de la formule (1.i), le terme d'intégrale stochastique par rapport à $W$ joue un rôle prépondérant. Précisément, on montre que

$$
\frac{1}{\log t} \sup _{s \leq t}\left|\Phi_{s}-\int_{0}^{s} \frac{d W_{u}}{W_{u}-f\left(Z_{u}\right)}\right| \underset{t \rightarrow \infty}{\stackrel{(P)}{\rightarrow}} 0 .
$$

Une fois ce résultat acquis, les raisonnements sont proches de ceux qui interviennent dans l'étude asymptotique des nombres de tours du mouvement brownien 
plan (voir [12]). On note $\beta+i \gamma$ le mouvement brownien complexe obtenu comme changé de temps de la martingale conforme (cf. Getoor-Sharpe [5]):

$$
\int_{0}^{t} \frac{d W_{s}}{W_{s}-f\left(Z_{s}\right)} \quad(t \geq 0)
$$

par l'inverse du processus croissant $\left(H_{t} ; t \geq 0\right)$ défini par

$$
H_{t}=\int_{0}^{t} \frac{d s}{\left|W_{s}-f\left(Z_{s}\right)\right|^{2}} .
$$

Exactement comme dans le cas du mouvement brownien plan (voir par exemple [12, Lemme 3.4]), on montre en utilisant (1.j) que, avec la notation $c=\frac{1}{2} \log t$,

$$
\frac{1}{c^{2}} H_{t}-T\left(\beta^{(c)}\right) \underset{t \rightarrow \infty}{\stackrel{(P)}{\rightarrow}} 0
$$

où $T\left(\beta^{(c)}\right)=\inf \left\{s ; \beta_{s}^{(c)}=1\right\}$ et on utilise la notation de "scaling" $\beta_{s}^{(c)}=$ $(1 / c) \beta_{c^{2} s}(s \geq 0)$. Pour conclure, on écrit maintenant

$$
\frac{1}{c} \operatorname{Im} \int_{0}^{t} \frac{d W_{s}}{W_{s}-f\left(Z_{s}\right)}=\frac{1}{c} \gamma_{H_{t}}=\gamma^{(c)}\left(\frac{1}{c^{2}} H_{t}\right)
$$

d'où, à l'aide de (1.j) et (1.k),

$$
\frac{2}{\log t} \theta_{t}-\gamma^{(c)}\left(T\left(\beta^{(c)}\right)\right) \underset{t \rightarrow \infty}{\stackrel{(P)}{\rightarrow}} 0,
$$

ce qui suffit pour établir (1.a) puisque, pour tout $c, \gamma^{(c)}\left(T\left(\beta^{(c)}\right)\right)$ suit une loi de Cauchy de paramètre 1.

Le point de départ de la preuve de (1.d) est le résultat limite (1.l) et son analogue pour $\left(\Gamma^{\prime}\right)$, qui s'écrit

$$
\frac{2}{\log t} \theta_{t}^{\prime}-\gamma^{\prime(c)}\left(T\left(\beta^{\prime(c)}\right)\right) \underset{t \rightarrow \infty}{\stackrel{(P)}{\rightarrow}} 0
$$

L'idée de la démonstration est de montrer ensuite:

$$
\left(\beta^{(c)}, \gamma^{(c)}, \beta^{(c)}, \gamma^{(c)}\right) \underset{c \rightarrow \infty}{\stackrel{(d)}{\rightrightarrows}}\left(\beta^{\infty}, \gamma^{\infty}, \beta^{\infty}, \gamma^{\prime \infty}\right)
$$

où $\left(\beta^{\infty}, \gamma^{\infty}, \beta^{\prime \infty}, \gamma^{\prime \infty}\right)$ est un quadruplet de mouvements browniens linéaires, dont la loi est décrite dans la partie 5. Il découle de (1.m) que

$$
\frac{2}{\log t}\left(\theta_{t}, \theta_{t}^{\prime}\right) \underset{t \rightarrow \infty}{\stackrel{(d)}{\rightarrow}}\left(\gamma^{\infty}\left(T\left(\beta^{\infty}\right)\right), \gamma^{\prime \infty}\left(T\left(\beta^{\prime \infty}\right)\right)\right)
$$

et il ne reste plus qu'à identifier la loi limite de (1.n) avec celle de (1.d). La partie difficile de la démonstration est la preuve de la convergence (1.m), et plus précisément l'identification de la loi du quadruplet limite $\left(\beta^{\infty}, \gamma^{\infty}, \beta^{\prime \infty}, \gamma^{\prime \infty}\right)$. Pour cela on utilise les critères démontrés dans [12] et [18] permettant d'établir que des mouvements browniens réels sont asymptotiquement indépendants, ou au contraire asymptotiquement identiques, en un sens qui sera précisé plus loin. 
Le but principal du présent travail est l'étude asymptotique de l'enlacement $\theta_{t}=\operatorname{Arg}\left(W_{t}-f\left(Z_{t}\right)\right)$. Cependant, les méthodes employées peuvent aisément être étendues à d'autres problèmes, par exemple à l'étude des petites valeurs de $\left|W_{t}-f\left(Z_{t}\right)\right|:$ voir [12, partie 6], pour une étude similaire, et la remarque de la fin de la partie 4 du présent article. Il est aussi facile d'adapter les méthodes de ce travail à l'étude asymptotique de $\operatorname{Arg}\left(W_{t}-F(t)\right)$, pour $F: \mathbf{R}_{+} \rightarrow \mathbf{R}^{2}$ fonction de classe $C^{1}$, ce qui revient à étudier le nombre de tours d'un mouvement brownien plan autour d'un point mobile. En résumé, bien que nous nous restreignions ici au problème spécifique des enlacements autour de courbes, les méthodes développées illustrent la puissance du calcul stochastique dans une vaste classe de problèmes asymptotiques (voir [18] pour d'autres applications).

La partie 2 est consacrée à quelques rappels concernant en particulier les critères d'indépendance, ou d'identité, asymptotique mentionnés ci-dessus. Dans la partie 3, nous établissons les résultats de convergence (1.j) qui jouent un rôle fondamental dans la suite. La preuve de ces résultats nécessite quelques estimations préliminaires qui présentent un certain intérêt en elles-mêmes. Dans la partie 4 , nous établissons, sous l'hypothèse $(\mathrm{H})$, le résultat de convergence (1.a), ainsi que quelques résultats auxiliaires qui seront utilisés dans la partie suivante. La partie 5 est consacrée à la preuve de (1.d). Enfin, dans la partie 6, nous calculons la transformée de Fourier de la loi limite de (1.d) et nous démontrons un lemme qui joue un rôle important dans la partie 5 .

\section{NOTATIONS ET RAPPELS}

(2.1) Soit $U=\left(U_{t}, t \geq 0\right)$ un processus à valeurs réelles. Pour tout $c>0$, nous désignons par $U^{(c)}=\left(U_{t}^{(c)}, t \geq 0\right)$ le processus déduit de $U$ par l'opération de scaling de rapport $c$, c'est à dire

$$
U_{t}^{(c)}=(1 / c) U_{c^{2} t} \quad(t \geq 0) .
$$

On utilisera fréquemment la notation $T(U)=\inf \left\{t ; U_{t}=1\right\}$.

(2.2) Soit $M=\left(M_{t}, t \geq 0\right)$ une martingale locale continue. Nous supposons que $\langle M\rangle_{\infty}=\infty$ p.s., ce qui sera toujours le cas dans nos applications. Il existe alors [1] un unique mouvement brownien réel $\eta=\left(\eta_{t}, t \geq 0\right)$ nul en 0 tel que pour tout $t \geq 0$ :

$$
M_{t}=M_{0}+\eta_{\langle M\rangle_{t}}
$$

Nous dirons que $\eta$ est le mouvement brownien associé à $M$. Le théorème suivant dû à Knight [9] donne une condition suffisante pour que les mouvements browniens associés à $n$ martingales locales continues soient indépendants.

Théorème 2.1. Soient $M^{1}, \ldots, M^{n} n$ martingales locales continues définies sur le même espace de probabilité filtré, et $\eta^{1}, \ldots, \eta^{n}$ les mouvements browniens associés respectivement à $M^{1}, \ldots, M^{n}$. Supposons que $\left\langle M^{i}\right\rangle_{\infty}=\infty$ p.s. $\quad(i=$ $1, \ldots, n)$ et que $\left\langle M^{i}, M^{j}\right\rangle \equiv 0 \quad(1 \leq i<j \leq n)$. Alors, $\eta^{1}, \ldots, \eta^{n}$ sont indépendants. 
Les hypothèses du Théorème 2.1 sont assez contraignantes puisqu'elles exigent que les crochets $\left\langle M^{i}, M^{j}\right\rangle$ soient identiquement nuls. La situation où les crochets $\left\langle M^{i}, M^{j}\right\rangle$ ne sont pas nuls mais néanmoins petits par rapport aux crochets $\left\langle M^{i}\right\rangle$ se présente assez souvent. On peut alors utiliser la version asymptotique suivante du théorème de Knight (voir [12, Théorème 4.1], [18, Theorem B.2], et les remarques qui suivent ce théorème).

Théorème 2.2. Soient $M^{1}, \ldots, M^{n} n$ martingales locales continues définies sur le même espace de probabilité filtré, telles que $\left\langle M^{i}\right\rangle_{\infty}=\infty$ pour tout $i=$ $1, \ldots, n$, et soient $\eta^{1}, \ldots, \eta^{n}$ les mouvements browniens associés respectivement à $M^{1}, \ldots, M^{n}$. Pour tout $i=1, \ldots, n$, soit $\tau^{i}=\left(\tau_{t}^{i}, t \geq 0\right)$ l'inverse continu à droite du processus croissant $\left\langle M^{i}\right\rangle$. Supposons que pour tout couple d'indices distincts $i, j \in\{1, \ldots, n\}$ on ait

$$
\frac{1}{t} \int_{0}^{\tau_{t}^{i}}\left|d\left\langle M^{i}, M^{j}\right\rangle_{s}\right| \underset{t \rightarrow \infty}{\stackrel{(P)}{\rightarrow}} 0 .
$$

Alors, les mouvements browniens $\eta^{1}, \ldots, \eta^{n}$ sont asymptotiquement indépendants, au sens suivant:

$$
\left(\eta^{1,(c)}, \ldots, \eta^{n,(c)}\right) \underset{c \rightarrow \infty}{\stackrel{(d)}{\rightarrow}}\left(\eta^{1, \infty}, \ldots, \eta^{n, \infty}\right)
$$

où $\eta^{1, \infty}, \ldots, \eta^{n, \infty}$ sont $n$ mouvements browniens indépendants.

Nous aurons aussi besoin de conditions suffisantes qui assurent que les mouvements browniens associés à deux martingales locales continues $M^{1}$ et $M^{2}$ sont asymptotiquement proches. Il suffira pour cela que le processus croissant $\left\langle M^{1}-M^{2}\right\rangle$. soit petit par rapport à $\left\langle M^{1}\right\rangle$. et $\left\langle M^{2}\right\rangle$. Précisément, on a le résultat suivant qui découle de la Proposition 4.3 de [12] (voir aussi [18, Theorem B.4]).

Théorème 2.3. Soient $M^{1}, M^{2}$ deux martingales locales continues définies sur le même espace de probabilité filtré, telles que $\left\langle M^{1}\right\rangle_{\infty}=\left\langle M^{2}\right\rangle_{\infty}=\infty$, et soient $\eta^{1}, \eta^{2}$ les mouvements browniens associés respectivement à $M^{1}, M^{2}$. Pour $i=1,2$, soit $\tau^{i}$. l'inverse continu à droite de $\left\langle M^{i}\right\rangle$. Supposons que

$$
\frac{1}{t}\left\langle M^{1}-M^{2}\right\rangle_{\tau_{t}^{1} \vee \tau_{t}^{2}} \stackrel{(P)}{\rightarrow} 0
$$

Alors $\eta^{1}$ et $\eta^{2}$ sont asymptotiquement identiques, au sens suivant: pour tout $T>0$

$$
\sup _{s \leq T}\left|\eta_{s}^{1,(c)}-\eta_{s}^{2,(c)}\right| \underset{c \rightarrow \infty}{\stackrel{(P)}{\rightarrow}} 0 .
$$

Remarque. Dans nos applications des Théorèmes 2.2 et 2.3, nous aurons fréquemment à considérer la situation où, pour tout $i=1, \ldots, n$,

$$
\frac{1}{\varphi(t)}\left\langle M^{i}\right\rangle_{t} \underset{t \rightarrow \infty}{\stackrel{(d)}{\rightarrow}} U
$$


où $\varphi$ est une fonction (déterministe) continue strictement çroissante de $\mathbf{R}_{+}$ dans $\mathbf{R}_{+}$et $U$ une variable aléatoire strictement positive p.s. On voit alors facilement que l'hypothèse du Théorème 2.2 est vérifiée, dès que, pour tout couple $(i, j)$ avec $i \neq j$,

$$
\frac{1}{\varphi(t)} \int_{0}^{t}\left|d\left\langle M^{i}, M^{j}\right\rangle_{s}\right| \underset{t \rightarrow \infty}{\stackrel{(P)}{\rightarrow}} 0 .
$$

De même, l'hypothèse du Théorème 2.3 est vérifiée par tout couple $\left(M^{i}, M^{j}\right)$ $(i \neq j)$ dès que

$$
\frac{1}{\varphi(t)}\left\langle M^{i}-M^{j}\right\rangle_{t} \stackrel{(P)}{\rightarrow} 0
$$

(2.3) Considérons maintenant $B=(X, Y, Z)$ mouvement brownien à valeurs dans $\mathbf{R}^{3}$. Le résultat suivant, dû à Dvoretzky et Erdös [4], décrit la vitesse à laquelle $\left|B_{t}\right|$ converge vers l'infini quand $t$ tend vers l'infini.

Théorème 2.4. Soit $g:] 0 ; \infty[\rightarrow] 0 ; \infty[$ une fonction décroissante. Une condition nécessaire et suffisante pour qu'on ait

$$
P \text { p.s., } \quad\left|B_{t}\right| \geq t^{1 / 2} g(t) \quad \text { pour tout } t \text { assez grand }
$$

est que

$$
\int_{1}^{\infty} g\left(e^{x}\right) d x<\infty
$$

Corollaire 2.5. Pour tout $\varepsilon>0$, on a

$$
\text { P p.s., } \quad\left|B_{t}\right| \geq t^{1 / 2}(\log t)^{-1-\varepsilon} \quad \text { pour tout } t \text { assez grand. }
$$

Comme dans l'introduction, notons $W_{t}=\left(X_{t}, Y_{t}\right) \quad(t \geq 0)$. Le résultat du corollaire nous servira surtout à dire que, pour $t$ assez grand, $\left|W_{t}\right|$ et $\left|Z_{t}\right|$ ne peuvent pas être simultanément petits. De manière plus précise, pour tout $0<b<1 / 2$,

$$
P \text { p.s. , pour } t \text { assez grand, soit }\left|W_{t}\right| \geq t^{b}, \text { soit }\left|Z_{t}\right| \geq t^{b} .
$$

\section{ESTIMATIONS PRELIMINAIRES}

(3.1) Nous nous proposons dans cette partie de montrer le résultat limite (1.j). On reprend les hypothèses et notations de l'introduction. Grâce à la formule (1.i), la preuve de (1.j) est ramenée à celle de la proposition suivante.

Proposition 3.1. Sous l'hypothèse $(\mathrm{H})$, on a

$$
\int_{0}^{\infty} \frac{\left|f^{\prime}\left(Z_{u}\right)\right|^{2} d u}{\left|W_{u}-f\left(Z_{u}\right)\right|^{2}}<\infty \quad P \text { p.s. }
$$

et

$$
\frac{1}{\log t} \sup _{s \leq t}\left|\int_{0}^{s} \frac{f^{\prime \prime}\left(Z_{u}\right) d u}{W_{u}-f\left(Z_{u}\right)}\right| \underset{t \rightarrow \infty}{\stackrel{(P)}{\rightarrow}} 0 .
$$


Remarquons que, en conséquence de la propriété (3.a), l'intégrale stochastique $\int_{0}^{t} f^{\prime}\left(Z_{u}\right) d Z_{u} /\left(W_{u}-f\left(Z_{u}\right)\right)$ converge p.s., lorsque $t \rightarrow \infty$. Nous établirons (3.a) en montrant le résultat un peu plus général suivant: si $g: \mathbf{R} \rightarrow \mathbf{R}^{2}$ est une fonction uniformément lipschitzienne sur tout $\mathbf{R}$, on a, pour tout $\alpha \in] 0 ; 1[$,

$$
\int_{1}^{\infty} \frac{d u}{\left|W_{u}-g\left(Z_{u}\right)\right|^{2}\left|Z_{u}\right|^{\alpha}}<\infty \text { p.s. }
$$

Compte-tenu de l'hypothèse $(\mathrm{H})$, on voit que (3.a) découle de (3.c), à condition de savoir que $\left(W_{u}-f\left(Z_{u}\right), u \geq 0\right)$ ne s'annule pas, ce qui découlera aussi de nos estimations.

La preuve de (3.b) pose certaines difficultés liées à la remarque suivante. Une majoration brutale du membre de gauche de (3.b) conduit à estimer

$$
\frac{1}{\log t} \int_{0}^{t} \frac{\left|f^{\prime \prime}\left(Z_{u}\right)\right|}{\left|W_{u}-f\left(Z_{u}\right)\right|} d u
$$

Cette majoration ne donne pas le résultat voulu. En effet, on peut montrer (nous n'utiliserons pas ce résultat) que

$$
\frac{1}{\log t} \int_{0}^{t} \frac{\left|f^{\prime \prime}\left(Z_{u}\right)\right|}{\left|W_{u}-f\left(Z_{u}\right)\right|} d u \underset{t \rightarrow \infty}{\stackrel{(P)}{\rightarrow}} \frac{1}{2} \int_{\mathbf{R}}\left|f^{\prime \prime}(z)\right| d z
$$

L'idée de la preuve de (3.b) sera de remplacer $f$ par une fonction $g$ coïncidant avec $f$ en dehors d'un compact mais telle que $\int\left|g^{\prime \prime}(z)\right| d z$ soit petite.

(3.2) Ce paragraphe est consacré à la démonstration de (3.c); les résultats obtenus serviront également à la démonstration de (3.b).

Proposition 3.2. Soit $g: \mathbf{R} \rightarrow \mathbf{R}^{2}$ une fonction lipschitzienne de rapport $K$ (i.e., $|g(x)-g(y)| \leq K|x-y|)$. Il existe une constante $C$, dépendant seulement de $K$, telle que, pour tout $0<\varepsilon<\frac{1}{2}$,

$$
P\left[\inf _{1 \leq s \leq 2}\left|W_{s}-g\left(Z_{s}\right)\right| \leq \varepsilon\right] \leq C\left(\log \frac{1}{\varepsilon}\right)^{-1} .
$$

Remarque. La proposition entraîne immédiatement:

$$
P\left[\inf \left\{\left|W_{s}-g\left(Z_{s}\right)\right| ; 1 \leq s \leq 2\right\}=0\right]=0 .
$$

Un changement d'échelle montre alors que pour tout entier $k \in \mathbf{Z}$,

$$
P\left[\inf \left\{\left|W_{s}-g\left(Z_{s}\right)\right| ; 2^{-k} \leq s \leq 2^{-k+1}\right\}=0\right]=0 .
$$

On en déduit que, Pp.s., il n'existe pas d'instant $t \in] 0 ; \infty\left[\right.$ tel que $W_{t}=g\left(Z_{t}\right)$ (ceci découle aussi du fait, facile à vérifier, que la courbe $w=g(z)$ est de capacité newtonienne nulle).

Lemme 3.3. Reprenons les hypothèses de la Proposition 3.1 et supposons de plus que, pour un $\varepsilon \in] 0 ; \frac{1}{2}\left[\right.$, on ait $\left|W_{0}-g\left(Z_{0}\right)\right|<\varepsilon$. Alors

$$
E\left[\int_{0}^{1} I\left(\left|W_{s}-g\left(Z_{s}\right)\right| \leq \varepsilon\right) d s\right] \geq \bar{C}^{2} \log \frac{1}{\varepsilon},
$$

pour une constante $\bar{C}>0$ dépendant seulement de $K$. 
Preuve. On se ramène aisément au cas où $W_{0}=w_{0}, Z_{0}=z_{0}$ avec $\left|w_{0}-g\left(z_{0}\right)\right|<\varepsilon$. Ensuite, on écrit

$$
\begin{aligned}
& E\left[\int_{0}^{1} I\left(\left|W_{s}-g\left(Z_{s}\right)\right| \leq \varepsilon\right) d s\right] \\
& \quad=\int_{0}^{1} d s \int_{\mathbf{R}} d z(2 \pi)^{-1 / 2} \exp \left(-z^{2} / 2\right) \\
& \quad \cdot \int_{\left\{\left|w_{0}+w-g\left(z_{0}+\sqrt{s} z\right)\right|<\varepsilon\right\}} d w(2 \pi s)^{-1} \exp \left(-|w|^{2} / 2 s\right) \\
& \quad \geq(2 \pi)^{-3 / 2} \int_{\varepsilon^{2}}^{1} \frac{d s}{s} \int_{-1}^{1} d z \exp \left(-z^{2} / 2\right) \varepsilon^{2} \exp \left(-\frac{(K \sqrt{s} z+2 \varepsilon)^{2}}{2 s}\right) \\
& \quad \geq(2 \pi)^{-3 / 2} \exp (-1 / 2) \exp \left(-K^{2}-4\right) \varepsilon^{2} \int_{\varepsilon^{2}}^{1} \frac{d s}{s} .
\end{aligned}
$$

Preuve de la Proposition 3.2. On remarque d'abord que pour $t \geq 1$ la densité de la loi de $W_{t}-g\left(Z_{t}\right)$ est majorée par $(2 \pi)^{-1}$, d'où

$$
E\left[\int_{1}^{3} I\left(\left|W_{s}-g\left(Z_{s}\right)\right| \leq \varepsilon\right) d s\right] \leq 2(2 \pi)^{-1} \varepsilon^{2} .
$$

Soit maintenant

$$
T_{\varepsilon}=\inf \left\{t \geq 1 ;\left|W_{t}-g\left(Z_{t}\right)\right| \leq \varepsilon\right\},
$$

avec la convention habituelle $\inf (\varnothing)=\infty$. En appliquant la propriété de Markov au temps $T_{\varepsilon}$ et en utilisant le Lemme 3.3, on trouve que

$$
E\left[\int_{1}^{3} I\left(\left|W_{s}-g\left(Z_{s}\right)\right| \leq \varepsilon\right) d s\right] \geq P\left(T_{\varepsilon} \leq 2\right) \bar{C} \varepsilon^{2} \log \left(\frac{1}{\varepsilon}\right)
$$

d'où aussi

$$
P\left(T_{\varepsilon} \leq 2\right) \leq\left(\bar{C} \varepsilon^{2} \log \frac{1}{\varepsilon}\right)^{-1} 2(2 \pi)^{-1} \varepsilon^{2}
$$

Remarque. Les techniques de la preuve de la Proposition 3.2 permettent facilement de montrer que, si $F$ est une fonction höldérienne d'exposant $1 / 2$ (i.e., $|F(t)-F(s)| \leq C|t-s|^{1 / 2}$ ) il n'existe p.s. pas d'instant $t>0$ tel que $W_{t}=F(t)$ (voir [11]).

Proposition 3.4. Sous les hypothèses de la Proposition 3.2, il existe une constante $C^{\prime}$ dépendant seulement de $K$ telle que, pour tout $r>0$,

$$
P\left[\int_{1}^{2} \frac{d s}{\left|W_{s}-g\left(Z_{s}\right)\right|^{2}}>r\right] \leq C^{\prime} r^{-1 / 3} .
$$

Preuve. Remarquons d'abord que

$$
\int_{1}^{2} \frac{d s}{\left|W_{s}-g\left(Z_{s}\right)\right|^{2}} \leq 1+\sum_{p=1}^{\infty} 2^{2 p} \int_{1}^{2} d s I\left(2^{-p} \leq\left|W_{s}-g\left(Z_{s}\right)\right| \leq 2^{-p+1}\right)
$$


et d'autre part, un calcul simple montre

$$
E\left[\int_{1}^{2} d s I\left(2^{-p} \leq\left|W_{s}-g\left(Z_{s}\right)\right| \leq 2^{-p+1}\right)\right] \leq 2.2^{-2 p} .
$$

En particulier, pour tout $r>2$,

$$
P\left[2^{2 p} \int_{1}^{2} d s I\left(2^{-p} \leq\left|W_{s}-g\left(Z_{s}\right)\right| \leq 2^{-p+1}\right)>r\right] \leq \frac{2}{r}
$$

d'où, en utilisant la Proposition 3.2,

$$
\begin{aligned}
P\left[\int_{1}^{2}\right. & \left.\frac{d s}{\left|W_{s}-g\left(Z_{s}\right)\right|^{2}}>r\right] \leq P\left[\inf \left\{\left|W_{s}-g\left(Z_{s}\right)\right| ; 1 \leq s \leq 2\right\} \leq 2^{-\left[r^{1 / 3}\right]}\right] \\
& +P\left[\sum_{p=1}^{\left[r^{1 / 3}\right]} 2^{2 p} \int_{1}^{2} I\left(2^{-p} \leq\left|W_{s}-g\left(Z_{s}\right)\right| \leq 2^{-p+1}\right) d s>r-1\right] \\
\leq & C\left((\log 2)\left[r^{1 / 3}\right]\right)^{-1} \\
& +\sum_{p=1}^{\left[r^{1 / 3}\right]} P\left[2^{2 p} \int_{1}^{2} I\left(2^{-p} \leq\left|W_{s}-g\left(Z_{s}\right)\right| \leq 2^{-p+1}\right) d s \geq\left[r^{1 / 3}\right]^{-1}(r-1)\right] \\
\leq & C\left((\log 2)\left[r^{1 / 3}\right]\right)^{-1}+2 \frac{\left[r^{1 / 3}\right]^{2}}{r-1} \\
\leq & C^{\prime} r^{-1 / 3} .
\end{aligned}
$$

Remarque. On peut certainement améliorer le résultat de la Proposition 3.4.

Dans le cas particulier $g=$ constante, on peut montrer que

$$
P\left[\int_{1}^{2} \frac{d s}{\left|W_{s}\right|^{2}}>r\right] \underset{r \rightarrow \infty}{\sim} C r^{-1 / 2} .
$$

Corollaire 3.5. Pour tout $0<\alpha<1$,

$$
\begin{aligned}
& \text { (i) } \int_{1}^{\infty} \frac{d s}{\left|W_{s}-g\left(Z_{s}\right)\right|^{2} s^{\alpha}}<\infty \quad P \text { p.s. } \\
& \text { (ii) } \int_{1}^{\infty} \frac{d s}{\left|W_{s}-g\left(Z_{s}\right)\right|^{2}\left|Z_{s}\right|^{\alpha}}<\infty \quad P \text { p.s. }
\end{aligned}
$$

Preuve. Commençons par montrer (i). On peut supposer $W_{0}=0, Z_{0}=0$. Alors

$$
\int_{1}^{\infty} \frac{d s}{\left|W_{s}-g\left(Z_{s}\right)\right|^{2} s^{\alpha}} \leq \sum_{p=0}^{\infty} 2^{-p \alpha} U_{p}
$$

où, pour tout $p \geq 0$, on note

$$
U_{p}=\int_{2^{p}}^{2^{p+1}} \frac{d s}{\left|W_{s}-g\left(Z_{s}\right)\right|^{2}} .
$$


Le changement de variables $s=2^{p} u$ montre que .

$$
U_{p} \stackrel{(d)}{=} \int_{1}^{2} \frac{d u}{\left|W_{u}-g_{p}\left(Z_{u}\right)\right|^{2}},
$$

où la fonction $g_{p}$ est définie par

$$
g_{p}(z)=2^{-p / 2} g\left(2^{p / 2} z\right)
$$

Remarquons que $g_{p}$ est lipschitzienne avec le même rapport que $g$. La Proposition 3.4 montre alors que pour tout $p \geq 0$ et tout $r>0$,

$$
P\left[U_{p}>r\right] \leq C r^{-1 / 3},
$$

où la constante $C$ ne dépend pas de $p$. Une application directe du lemme de Borel-Cantelli montre que, pour tout $\beta>3, P$ p.s., pour $p$ assez grand, $U_{p} \leq p^{\beta}$, ce qui entraîne que la série $\sum 2^{-p \alpha} U_{p}$ converge.

Nous passons maintenant à la preuve de (ii). On utilise la remarque (2.b) suivant le Corollaire 2.5. Si $0<b<b^{\prime}<\frac{1}{2}$, on a pour $t$ assez grand, soit $\left|Z_{t}\right| \geq t^{b}$, soit $\left|Z_{t}\right|<t^{b}$ et alors $\left|W_{t}\right| \geq t^{b^{\prime}}$. Dans le second cas, compte-tenu du caractère lipschitzien de $g$ on a, toujours pour $t$ grand,

$$
\left|W_{t}-g\left(Z_{t}\right)\right| \geq t^{b^{\prime}}-\left(\alpha+\beta t^{b}\right) \geq \frac{1}{2} t^{b^{\prime}} .
$$

On voit donc que, pour montrer (ii), il suffit d'établir la finitude des deux intégrales:

$$
\int_{1}^{\infty} \frac{d s}{\left|W_{s}-g\left(Z_{s}\right)\right|^{2} s^{\alpha b}} \quad \text { et } \quad \int_{1}^{\infty} \frac{d s}{s^{2 b^{\prime}}\left|Z_{s}\right|^{\alpha}} .
$$

Or, pour la première, cela découle da la partie (i) du corollaire, et, pour la seconde, on remarque que, si $b^{\prime}$ est choisi tel que $2 b^{\prime}+\alpha / 2>1$, on a

$$
E\left[\int_{1}^{\infty} \frac{d s}{s^{2 b^{\prime}}\left|Z_{s}\right|^{\alpha}}\right] \leq C \int_{1}^{\infty} \frac{d s}{s^{2 b^{\prime}+\alpha / 2}}<\infty .
$$

La première assertion de la Proposition 3.1 découle du Corollaire 2.5 et de la remarque suivant la Proposition 3.1. La seconde assertion sera établie dans le sous-paragraphe suivant.

Remarque. Ici encore, on peut sensiblement améliorer les résultats du corollaire. Par exemple, la preuve ci-dessus montre que pour tout $\beta>4$,

$$
\int^{\infty} \frac{d s}{\left|W_{s}-g\left(Z_{s}\right)\right|^{2}(\log s)^{\beta}}<\infty \text { p.s. }
$$

(3.3) Nous allons maintenant montrer (3.b) en utilisant les résultats précédents. On remarque d'abord que

$$
\begin{aligned}
\sup _{1 \leq s \leq t}\left|\int_{1}^{s} \frac{f^{\prime \prime}\left(Z_{u}\right) d u}{W_{u}-f\left(Z_{u}\right)}\right| & \leq \int_{1}^{t} \frac{\left|f^{\prime \prime}\left(Z_{u}\right)\right| d u}{\left|W_{u}-f\left(Z_{u}\right)\right|} \\
& =\int_{\mathbf{R}}\left|f^{\prime \prime}(z)\right| d z \int_{1}^{t} \frac{1}{\left|W_{u}-f(z)\right|} d_{u} L_{u}^{z}(Z) .
\end{aligned}
$$


Ici, $\int \phi(u) d_{u} L_{u}^{z}(Z)$ désigne l'intégrale de Stieltjes de $\phi$ par rapport au temps local $\left(L_{u}^{z}(Z), u \geq 0\right)$ de $Z$ au niveau $z$. Compte-tenu de l'indépendance de $W$ et $Z$, on a

$$
\begin{aligned}
E\left[\int_{1}^{t} \frac{1}{\left|W_{u}-f(z)\right|} d_{u} L_{u}^{z}(Z)\right] & =\int_{1}^{t} E\left(\frac{1}{\left|W_{u}-f(z)\right|}\right) d_{u} E\left(L_{u}^{z}(Z)\right) \\
& =\int_{1}^{t} E\left(\frac{1}{\left|W_{u}-f(z)\right|}\right) \frac{d u}{\sqrt{2 \pi u}} \exp -\frac{\left(z-z_{0}\right)^{2}}{2 u} \\
& \leq C \int_{1}^{t} \frac{d u}{u}=C \log t
\end{aligned}
$$

On a donc montré

$$
\frac{1}{\log t} E\left[\sup _{s \leq t}\left|\int_{0}^{s} \frac{f^{\prime \prime}\left(Z_{u}\right) d u}{W_{u}-f\left(Z_{u}\right)}\right|\right] \leq C \int_{\mathbf{R}}\left|f^{\prime \prime}(z)\right| d z
$$

pour une certaine constante $C$ ne dépendant pas de $f$.

Revenons maintenant à la formule (1.i)

$$
\Phi_{t}-\Phi_{0}=\int_{0}^{t} \frac{d W_{u}}{W_{u}-f\left(Z_{u}\right)}+R_{t}-\frac{1}{2} \int_{0}^{t} \frac{f^{\prime \prime}\left(Z_{u}\right) d u}{W_{u}-f\left(Z_{u}\right)},
$$

où on a noté

$$
R_{t}=-\int_{0}^{t} \frac{f^{\prime}\left(Z_{u}\right) d Z_{u}}{W_{u}-f\left(Z_{u}\right)}-\frac{1}{2} \int_{0}^{t}\left(\frac{f^{\prime}\left(Z_{u}\right)}{W_{u}-f\left(Z_{u}\right)}\right)^{2} d u
$$

(3.a) entraîne

$$
R_{t} \underset{t \rightarrow \infty}{\stackrel{\text { p.s. }}{\rightarrow}} R_{\infty} .
$$

Soit maintenant $K>0$ une constante dont le choix sera précisé plus loin. On introduit une fonction auxiliaire $g$ définie de la manière suivante: $g$ coïncide avec $f$ sur le complémentaire de $]-K, K$ [ et $g$ est affine sur $[-K, K]$. La fonction $g$ n'est pas de classe $C^{2}$ mais sa dérivée seconde, au sens des distributions, est une mesure de Radon, et on peut donc appliquer la formule d'Itô généralisée pour développer $\left(\Phi_{t}^{\prime} ; t \geq 0\right)$, détermination continue du logarithme le long de la courbe $\left(W_{t}-g\left(Z_{t}\right) ; t \geq 0\right)$, au moyen de la formule (1.i). Ceci nous conduit à

$$
\Phi_{t}^{\prime}-\Phi_{0}^{\prime}=\int_{0}^{t} \frac{d W_{u}}{W_{u}-g\left(Z_{u}\right)}+R_{t}^{\prime}+\frac{1}{2} \int_{\mathbf{R}} g^{\prime \prime}(d z) \int_{0}^{t} \frac{d_{s} L_{s}^{z}(Z)}{W_{s}-g(z)},
$$

où pour les mêmes raisons que précédemment

$$
R_{t}^{\prime} \underset{t \rightarrow \infty}{\stackrel{\text { p.s. }}{\rightarrow}} R_{\infty}^{\prime} .
$$

Il est clair que

$$
\Phi_{t}-\Phi_{t}^{\prime} \underset{t \rightarrow \infty}{\stackrel{\text { p.s. }}{\rightarrow}} .
$$


De plus, si l'on note

$$
M_{t}=\int_{0}^{t} \frac{d W_{u}}{W_{u}-f\left(Z_{u}\right)} \quad \text { et } \quad M_{t}^{\prime}=\int_{0}^{t} \frac{d W_{u}}{W_{u}-g\left(Z_{u}\right)},
$$

on a aussi

$$
M_{t}-M_{t}^{\prime} \underset{t \rightarrow \infty}{\stackrel{\text { p.s. }}{\rightarrow}} \text {. }
$$

Ceci est une conséquence du Corollaire 3.5(ii), qui entraîne que

$$
\left\langle M-M^{\prime}, \bar{M}-\bar{M}^{\prime}\right\rangle_{\infty}=2 \int_{0}^{\infty} d u\left|\frac{1}{W_{u}-f\left(Z_{u}\right)}-\frac{1}{W_{u}-g\left(Z_{u}\right)}\right|^{2}<\infty, \quad \text { p.s. }
$$

Il découle de ces remarques et de (3.e), (3.e' ) que

$$
\int_{0}^{t} \frac{f^{\prime \prime}\left(Z_{s}\right) d s}{W_{s}-f\left(Z_{s}\right)}-\int_{\mathbf{R}} g^{\prime \prime}(d z) \int_{0}^{t} \frac{1}{W_{s}-g(z)} d_{s} L_{s}^{z}(Z) \underset{t \rightarrow \infty}{\stackrel{\text { p.s. }}{\rightarrow}} .
$$

Or, comme ci-dessus

$$
\frac{1}{\log t} E\left[\sup _{u \leq t}\left|\int_{\mathbf{R}} g^{\prime \prime}(d z) \int_{0}^{u} \frac{d_{s} L_{s}^{z}(Z)}{W_{s}-g(z)}\right|\right] \leq C \int_{\mathbf{R}}\left|g^{\prime \prime}(d z)\right|
$$

et, grâce à l'hypothèse $(\mathrm{H})$, on peut, pour tout $\varepsilon>0$, choisir $K$ assez grand de façon que

$$
C \int\left|g^{\prime \prime}(d z)\right|<\varepsilon
$$

On conclut finalement que

$$
\frac{1}{\log t} \sup _{s \leq t}\left|\int_{0}^{s} \frac{f^{\prime \prime}\left(Z_{u}\right) d u}{W_{u}-f\left(Z_{u}\right)}\right| \underset{t \rightarrow \infty}{\stackrel{(P)}{\rightarrow}} 0
$$

ce qui termine la preuve de (3.b) et de la Proposition 3.1.

\section{ENLACEMENTS AUtour D'UNe COURBE}

(4.1) Nous nous proposons dans cette partie d'établir le résultat limite (1.a) en nous appuyant sur les estimations de la partie 3 . On considère une fonction $f$ satisfaisant l'hypothèse $(\mathrm{H})$ de l'introduction et on reprend les notations de la partie 1 , en particulier:

$$
H_{t}=\int_{0}^{t} \frac{d s}{\left|W_{s}-f\left(Z_{s}\right)\right|^{2}}
$$

et $\beta$ désigne le mouvement brownien associé à la martingale

$$
\operatorname{Re} \int_{0}^{t} \frac{d W_{s}}{W_{s}-f\left(Z_{s}\right)}
$$

On note aussi $\rho_{t}=\operatorname{Re} \Phi_{t}=\log \left|W_{t}-f\left(Z_{t}\right)\right|$, de sorte que $\Phi_{t}=\rho_{t}+i \theta_{t}$. 
Lemme 4.1. Notons $c=\frac{1}{2} \log t$. Alors

$$
\frac{1}{c^{2}} H_{t}-T\left(\beta^{(c)}\right) \underset{t \rightarrow \infty}{\stackrel{(P)}{\rightarrow}} 0
$$

Preuve. Soit $\left(\tau_{s}, s \geq 0\right)$ l'inverse de $H$. En appliquant (1.i) avec $t=\tau_{s}$, on trouve

$$
\rho_{\tau_{s}}=\beta_{s}+\Sigma_{\tau_{s}}
$$

où, d'après la Proposition 3.1,

$$
\frac{1}{\log t} \sup \left\{\left|\Sigma_{s}\right|, s \leq t\right\} \underset{t \rightarrow \infty}{\stackrel{(P)}{\rightarrow}} 0 .
$$

D'autre part,

$$
\begin{aligned}
H_{t} & =\inf \left\{u ; \int_{0}^{u} d s \mid W_{\tau_{s}}-f\left(\left.Z_{\tau_{s}}\right|^{2}>t\right\}\right. \\
& =\inf \left\{u ; \int_{0}^{u} d s \exp 2\left(\beta_{s}+\Sigma_{\tau_{s}}\right)>t\right\} .
\end{aligned}
$$

Notant $c=\frac{1}{2} \log t$, on trouve

$$
\begin{aligned}
\frac{1}{c^{2}} H_{t} & =\inf \left\{u ; \int_{0}^{c^{2} u} d s \exp 2\left(\beta_{s}+\Sigma_{\tau_{s}}\right)>t\right\} \\
& =\inf \left\{u ; c^{2} \int_{0}^{u} d s \exp 2 c\left(\beta_{s}^{(c)}+\frac{1}{c} \Sigma_{\tau_{c^{2} s}}\right)>t\right\} \\
& =\inf \left\{u ; \frac{1}{2 c} \log \int_{0}^{u} d s \exp 2 c\left(\beta_{s}^{(c)}+\frac{1}{c} \Sigma_{\tau_{c^{2} s}}\right)>1-\frac{\log c}{c}\right\} .
\end{aligned}
$$

Admettons provisoirement que, pour tout $T \geq 0$,

$$
\frac{1}{c} \sup \left(\left|\Sigma_{\tau_{c^{2} s}}\right| ; s \leq T\right) \underset{c \rightarrow \infty}{\stackrel{(P)}{\rightarrow}} 0 .
$$

On vérifie alors que pour tout $T>0$,

$$
\frac{1}{2 c} \log \left[\int_{0}^{T} d s \exp 2 c\left(\beta_{s}^{(c)}+\frac{1}{c} \Sigma_{\tau^{2} s}\right)\right]-\sup _{s \leq T} \beta_{s}^{(c)} \underset{c \rightarrow \infty}{\stackrel{(P)}{\rightarrow}} 0,
$$

d'où aisément

$$
\frac{1}{c^{2}} H_{t}-\inf \left\{s ; \beta_{s}^{(c)}=1\right\} \underset{t \rightarrow \infty}{\stackrel{(P)}{\rightarrow}} 0 .
$$

Il reste à montrer (4.b). On sait déjà que

$$
\frac{1}{\log t} \sup \left(\left|\Sigma_{s}\right| ; s \leq t\right) \underset{t \rightarrow \infty}{\stackrel{(P)}{\rightarrow}} 0 .
$$

Compte-tenu de ce résultat, il suffit pour établir (4.b) de montrer que

$$
\lim _{K \rightarrow+\infty}\left(\limsup _{c \rightarrow \infty} P\left[\frac{1}{c} \log \tau_{c^{2} T}>K\right]\right)=0 .
$$


En effet, si on admet (4.b), on peut, pour tout $\varepsilon>0$, choisir $K>0$ de façon que, pour tout $c$ assez grand,

$$
P\left[\tau_{c^{2} T}>e^{K c}\right]<\varepsilon .
$$

D'autre part, sur l'ensemble $\left\{\tau_{c^{2} T} \leq e^{K c}\right\}$,

$$
\frac{1}{c} \sup _{s \leq T}\left|\Sigma_{\tau_{c^{2} s}}\right| \leq \frac{1}{c} \sup _{t \leq e^{K c}}\left|\Sigma_{t}\right| \underset{c \rightarrow \infty}{\stackrel{(P)}{\rightarrow}} 0
$$

d'après (4.a).

Pour montrer (4.c), on remarque d'abord que

$$
P\left[\frac{1}{c} \log \tau_{c^{2} T}>K\right]=P\left[H_{e^{K c}}<c^{2} T\right]=P\left[H_{u(c)}<\frac{T}{K^{2}}(\log u(c))^{2}\right],
$$

où on a noté $u(c)=e^{K c}$. L'identité (4.c) découle donc de

$$
\lim _{\varepsilon \rightarrow 0}\left(\limsup _{u \rightarrow \infty} P\left[H_{u}<\varepsilon(\log u)^{2}\right]\right)=0 .
$$

Pour vérifier cette dernière égalité, on utilise la majoration $|f(z)| \leq c\left(1+|z|^{\delta}\right)$ (pour un $\delta<1$ ); ainsi

$$
H_{t}=\int_{0}^{t} \frac{d s}{\left|W_{s}-f\left(Z_{s}\right)\right|^{2}} \geq \frac{1}{4} \int_{0}^{t} \frac{d s}{\left|W_{s}\right|^{2}} I\left(\left|W_{s}\right| \geq 2 c\left(1+\left|Z_{s}\right|^{\delta}\right)\right)
$$

et, d'après la Proposition 4 de [15],

$$
(\log t)^{-2} \int_{0}^{t} \frac{d s}{\left|W_{s}\right|^{2}} I\left(\left|W_{s}\right| \geq 2 c\left(1+\left|Z_{s}\right|^{\delta}\right)\right) \underset{t \rightarrow \infty}{\stackrel{(d)}{\rightarrow}} \frac{1}{4} \int_{0}^{T(\eta)} d s I\left(\eta_{s} \geq \delta \hat{\eta}_{s}\right),
$$

où $\eta=\left(\eta_{t}, t \geq 0\right)$ désigne un mouvement brownien réel issu de $0, \hat{\eta}_{t}=$ $\sup \left\{\eta_{s} ; s \leq t\right\}$ et $T(\eta)=\inf \left\{t ; \eta_{t}=1\right\}$. Comme la variable limite ci-dessus est presque sûrement strictement positive, on en déduit (4.d). Ceci termine la preuve du lemme.

Théorème 4.2. Sous l'hypothèse $(\mathrm{H})$, on a

$$
\frac{2}{\log t} \theta_{t} \underset{t \rightarrow \infty}{\stackrel{(d)}{\rightarrow}} C_{1}
$$

où $C_{1}$ est une variable de Cauchy de paramètre 1 .

Preuve. Comme dans l'introduction, soit $\beta+i \gamma$ le mouvement brownien complexe associé à la martingale conforme $\int_{0}^{t} d W_{s} /\left(W_{s}-f\left(Z_{s}\right)\right)$. Alors, d'après (1.i) et la Proposition 3.1,

$$
\theta_{t}=\gamma_{H_{t}}+\Omega_{t}
$$

où

$$
\frac{1}{\log t} \sup \left\{\left|\Omega_{s}\right| ; s \leq t\right\} \underset{t \rightarrow \infty}{\stackrel{(P)}{\rightarrow}} 0
$$


Notant $c=\frac{1}{2} \log t$, on trouve

$$
\frac{1}{c} \theta_{t}-\frac{1}{c} \gamma_{H_{t}} \underset{t \rightarrow \infty}{\stackrel{(P)}{\rightarrow}} 0
$$

D'autre part,

$$
\frac{1}{c} \gamma_{H_{t}}=\gamma^{(c)}\left(H_{t} / c^{2}\right)
$$

et, d'après le Lemme 4.1 ,

$$
\gamma^{(c)}\left(H_{t} / c^{2}\right)-\gamma^{(c)}\left(T\left(\beta^{(c)}\right)\right) \underset{t \rightarrow \infty}{\stackrel{(P)}{\rightarrow}} 0 .
$$

Ceci termine la preuve du théorème puisque pour tout $c, \gamma^{(c)}\left(T\left(\beta^{(c)}\right)\right)$ suit une loi de Cauchy de paramètre 1 .

(4.2) Au cours de la preuve du Lemme 4.1, nous avons établi un certain nombre de résultats qui présentent de l'intérêt en eux-mêmes et nous seront utiles dans la suite. L'objet de la proposition suivante est de regrouper et de préciser ces résultats.

\section{Proposition 4.3.}

(i) Pour tout $s>0$, si $\hat{\beta}_{s}^{(c)}=\sup \left\{\beta_{u}^{(c)} ; u \leq s\right\}$,

$$
\frac{1}{2 c} \log \left(\tau_{c^{2} s}\right)-\hat{\beta}_{s}^{(c)} \underset{c \rightarrow \infty}{\stackrel{(P)}{\rightarrow}} 0
$$

(ii) Notons $c=\frac{1}{2} \log t$. Alors, pour tous $-\infty \leq \alpha \leq \bar{\alpha} \leq \infty$,

$$
\begin{aligned}
\frac{1}{c^{2}} \int_{0}^{t} \frac{d s}{\left|W_{s}-f\left(Z_{s}\right)\right|^{2}} & I\left(s^{\alpha} \leq\left|W_{s}-f\left(Z_{s}\right)\right| \leq s^{\bar{\alpha}}\right) \\
& -\int_{0}^{T\left(\beta^{(c)}\right)} d u I\left(2 \alpha \hat{\beta}_{u}^{(c)} \leq \beta_{u}^{(c)} \leq 2 \bar{\alpha} \hat{\beta}_{u}^{(c)}\right)
\end{aligned}
$$

converge en probabilité vers 0 lorsque $t \rightarrow \infty$.

Preuve. L'assertion (i) a déjà été utilisée dans la preuve du Lemme 4.1; elle découle des identités

$$
\begin{aligned}
\frac{1}{c} \log \left(\tau_{c^{2} s}\right) & =\frac{1}{2 c} \log \left(\int_{0}^{c^{2} s} \exp \left(2 \rho_{u}\right) d u\right) \\
& =\frac{1}{2 c} \log \left(\int_{0}^{s} d u \exp 2 c\left(\beta_{u}^{(c)}+\frac{1}{c} \Sigma_{\tau_{c^{2} u}}\right)\right)+\frac{\log c}{c}
\end{aligned}
$$

et du résultat limite (4.b). Pour montrer (ii), on écrit

$$
\begin{aligned}
& \frac{4}{(\log t)^{2}} \int_{0}^{t} \frac{d s}{\left|W_{s}-f\left(Z_{s}\right)\right|^{2}} I\left(s^{\alpha} \leq\left|W_{s}-f\left(Z_{s}\right)\right| \leq s^{\bar{\alpha}}\right) \\
& =\frac{1}{c^{2}} \int_{0}^{H_{t}} d u I\left(\alpha \log \tau_{u} \leq \rho_{\tau_{u}} \leq \bar{\alpha} \log \tau_{u}\right) \\
& =\int_{0}^{H_{t} / c^{2}} d u I\left(\frac{\alpha}{c} \log \tau_{c^{2} u} \leq \frac{1}{c} \rho_{\tau_{c^{2} u}} \leq \frac{\bar{\alpha}}{c} \log \tau_{c^{2} u}\right) .
\end{aligned}
$$


On peut d'abord utiliser le Lemme 4.1 pour remplacer $H_{t} / c^{2}$ par $T\left(\beta^{(c)}\right)$. Ensuite, comme on l'a déjà remarqué,

$$
\frac{1}{c} \rho_{\tau_{c^{2} u}}-\beta_{u}^{(c)} \underset{c \rightarrow \infty}{\stackrel{(P)}{\rightarrow}} 0
$$

et on conclut en utilisant l'assertion (i).

Remarque. Les méthodes développées ci-dessus pour l'étude du nombre de tours du mouvement brownien $B$ autour de la courbe $(\Gamma)$ peuvent aussi être appliquées à des théorèmes limites concernant la vitesse d'approche de cette courbe par le mouvement brownien. Précisément, on pose, pour tout $r>0$,

$$
T_{r}=\inf \left\{t \geq 0 ;\left|W_{t}-g\left(Z_{t}\right)\right|<r\right\}
$$

et on s'intéresse au comportement asymptotique de $T_{r}$ quand $r$ tend vers 0 . Les arguments développés en [12, partie 6], permettent de déduire du Lemme 4.1 que, toujours sous l'hypothèse $(\mathrm{H})$,

$$
\frac{\log T_{r}}{|\log r|} \underset{r \rightarrow 0}{\stackrel{(d)}{\rightarrow}} 2 \frac{e}{e^{\prime}}
$$

où $e$ et $e^{\prime}$ sont deux variables exponentielles standard indépendantes.

\section{LOI ASYMPTOTIQUE DES ENLACEMENTS AUTOUR DE DEUX COURBES}

(5.1) Dans cette partie, nous nous proposons d'obtenir la loi asymptotique des processus d'enlacement du mouvement brownien $B$ autour de deux courbes de l'espace. Nous considérons deux fonctions $f$ et $g$ satisfaisant l'hypothèse $(\mathrm{H})$ de l'introduction; $\theta$, respectivement $\theta^{\prime}$, désigne le processus d'enlacement de $B$ autour de la courbe $(x, y)=f(z)$, respectivement la courbe $(x, y)=g(z)$.

Théorème 5.1. Supposons qu'il existe $a \in[-\infty, 1[$ tel que

$$
\lim _{|z| \rightarrow \infty} \frac{1}{\log |z|} \log |f(z)-g(z)|=a .
$$

Alors,

$$
\frac{2}{\log t}\left(\theta_{t}, \theta_{t}^{\prime}\right) \underset{t \rightarrow \infty}{\stackrel{(d)}{\rightarrow}}\left(\omega_{1}\left(\sigma_{1}\right)+\omega_{2}\left(\sigma_{2}\right), \omega_{1}^{\prime}\left(\sigma_{1}^{\prime}\right)+\omega_{2}\left(\sigma_{2}\right)\right)
$$

où $\left(\omega_{1}, \omega_{1}^{\prime}, \omega_{2}\right)$ est un triplet de mouvements browniens réels indépendants issus de 0 , indépendant du triplet $\left(\sigma_{1}, \sigma_{1}^{\prime}, \sigma_{2}\right)$, dont la loi peut être décrite comme suit: soient $\eta=\left(\eta_{t}, t \geq 0\right)$ un mouvement brownien réel issu de $0, \hat{\eta}_{t}=\sup \left\{\eta_{s} ; s \leq t\right\}$ et $T(\eta)=\inf \left\{t ; \eta_{t}=1\right\}$; soit $l_{T(\eta)}^{(a)}$ le temps local en 0 à l'instant $T(\eta) d u$ processus $\eta$-âे (on convient que $l_{T(\eta)}^{(a)}=0$ si $a=-\infty$ ). Alors,

$$
\left(\sigma_{1}, \sigma_{1}^{\prime}, \sigma_{2}\right) \stackrel{(d)}{=}\left(\frac{1}{4}\left(l_{T(\eta)}^{(a)}\right)^{2} T_{1}, \frac{1}{4}\left(l_{T(\eta)}^{(a)}\right)^{2} T_{2}, \int_{0}^{T(\eta)} I\left(\eta_{s} \geq a \hat{\eta}_{s}\right) d s\right),
$$


où $T_{1}, T_{2}$ sont deux variables indépendantes et indépendantes de $\eta$, de même loi que $T(\eta)$. De façon équivalente, pour tous $\lambda_{1}, \lambda_{1}^{\prime}, \lambda_{2} \in \mathbf{R}$,

$$
\begin{gathered}
E\left[\exp i\left(\lambda_{1} \omega_{1}\left(\sigma_{1}\right)+\lambda_{1}^{\prime} \omega_{1}^{\prime}\left(\sigma_{1}^{\prime}\right)+\lambda_{2} \omega_{2}\left(\sigma_{2}\right)\right)\right] \\
=\left(\operatorname{ch} \lambda_{2} \bar{a}+\left(\left|\lambda_{1}\right|+\left|\lambda_{1}^{\prime}\right|\right) \frac{\operatorname{sh} \lambda_{2} \bar{a}}{\lambda_{2}}\right)^{-1 / \bar{a}},
\end{gathered}
$$

où $\bar{a}=1-a$ (dans le cas $a=-\infty$, la formule (5.c) doit être interprétée comme: $\left.E\left[\exp i \lambda_{2} \omega_{2}\left(\sigma_{2}\right)\right]=\exp -\left|\lambda_{2}\right|\right)$.

Remarques. (i) La loi du triplet $\left(\sigma_{1}, \sigma_{1}^{\prime}, \sigma_{2}\right)$ est étudiée dans la partie 6 , où est démontrée la formule (5.c). Remarquons ici que la loi limite du théorème généralise celle qu'on obtient dans l'étude des nombres de tours du mouvement brownien plan autour de deux points (voir [18]), qu'on retrouve ici lorsque $a=0$.

(ii) Le terme $\omega_{2}\left(\sigma_{2}\right)$ correspond ici aux "enlacements communs" autour des deux courbes considérées. On retrouve ainsi une décomposition très semblable à la décomposition en "grand angle" et "petit angle" pour les nombres de tours du mouvement brownien plan (voir [12, 16, 18]).

(iii) Un cas particulier intéressant est celui où $g \equiv 0$ et où $a<0$. La courbe $(x, y)=f(z)$ admet alors pour asymptote la droite $x=y=0$. Le théorème permet de comparer les nombres de tours du mouvement brownien autour de la courbe et de son asymptote.

Pour éviter certaines banalités, nous donnerons la preuve du Théorème 5.1 seulement dans le cas $a>-\infty$. Nous laisserons au lecteur le soin de vérifier que les arguments utilisés s'appliqueraient aussi, sous une forme plus simple, au cas $a=-\infty$. La preuve du Théorème 5.1 utilise un lemme, intéressant en luimême, concernant certaines fonctionnelles du mouvement brownien linéaire, et que nous démontrerons dans la partie 6 .

Lemme 5.2. Soient $\eta=\left(\eta_{t}, t \geq 0\right)$ un mouvement brownien réel issu de $\varepsilon \in$ ]0; $1\left[, \hat{\eta}_{t}=\sup \left\{\eta_{s}, s \leq t\right\}\right.$ et $T(\eta)=\inf \left\{t, \eta_{t}=1\right\}$. Soient $\left.a \in\right]-\infty, 1[$ et $\underline{\eta}$, resp. $\bar{\eta}$ le mouvement brownien associé à la martingale continue

$$
\int_{0}^{t} I\left(\eta_{s}<a \hat{\eta}_{s}\right) d \eta_{s}, \quad \text { resp. } \quad \int_{0}^{t} I\left(\eta_{s} \geq a \hat{\eta}_{s}\right) d \eta_{s} .
$$

Alors,

(i) si $\mathscr{F}^{\eta}$, resp. $\mathscr{F} \underline{\eta}, \mathscr{F}^{\bar{\eta}}$ désigne la tribu engendrée par $\eta$, resp. $\underline{\eta}, \bar{\eta}$, les tribus $\mathscr{F}^{\underline{\eta}}$ et $\mathscr{F}^{\bar{\eta}}$ sont indépendantes, et $\mathscr{F}^{\eta}=\mathscr{F}^{\underline{\eta}} \vee \mathscr{F}^{\bar{\eta}}$;

(ii) soient

$$
\sigma_{1}=\int_{0}^{T(\eta)} I\left(\eta_{s}<a \hat{\eta}_{s}\right) d s ; \quad \sigma_{2}=\int_{0}^{T(\eta)} I\left(\eta_{s} \geq a \hat{\eta}_{s}\right) d s
$$

et soit $l_{T(\eta)}^{(a)}$ le temps local en 0 , à l'instant $T(\eta)$ du processus $\eta$-âे; alors, $\sigma_{2}$ et $l_{T(\eta)}^{(a)}$ sont mesurables par rapport à $\mathscr{F}^{\bar{\eta}}$, et

$$
\sigma_{1}=\inf \left\{t ; \underline{\eta}_{t}=\frac{1}{2} l_{T(\eta)}^{(a)}\right\}
$$


(5.2) Notations. Nous reprenons les notations $\rho, \beta, \gamma$ introduites dans les parties précédentes pour désigner certains processus intervenant dans l'étude de $W_{t}-f\left(Z_{t}\right)$. Les processus analogues correspondant à $W_{t}-g\left(Z_{t}\right)$ sont notés $\rho^{\prime}, \beta^{\prime}, \gamma^{\prime}$. On introduit en outre le mouvement brownien complexe $\bar{\beta}+i \bar{\gamma}$, resp. $\underline{\beta}+i \underline{\gamma}$, associé à la martingale conforme

$$
\bar{M}_{t}+i \bar{N}_{t}=\int_{0}^{t} \frac{d W_{s}}{W_{s}-f\left(Z_{s}\right)} I\left(\left|W_{s}-f\left(Z_{s}\right)\right| \geq s^{a / 2}\right)
$$

resp. à

$$
\underline{M}_{t}+i \underline{N}_{t}=\int_{0}^{t} \frac{d W_{s}}{W_{s}-f\left(Z_{s}\right)} I\left(\left|W_{s}-f\left(Z_{s}\right)\right|<s^{a / 2}\right) .
$$

On définit de même $\bar{\beta}^{\prime}, \underline{\beta^{\prime}}, \bar{\gamma}^{\prime}, \underline{\gamma}^{\prime}, \bar{M}^{\prime}, \underline{M^{\prime}}, \bar{N}^{\prime}, \underline{N^{\prime}}$ en remplaçant $f$ par $g$.

La preuve du Théorème 5.1 repose sur le lemme-clé suivant.

Lemme 5.3. Supposons qu'on ait, le long d'une suite $\left(c_{n}\right)$ croissant vers $+\infty$,

$$
\begin{aligned}
& \left(\beta^{(c)}, \bar{\beta}^{(c)}, \underline{\beta}^{(c)}, \beta^{\prime(c)}, \bar{\beta}^{\prime(c)}, \underline{\beta}^{(c)}, \gamma^{(c)}, \bar{\gamma}^{(c)}, \underline{\gamma}^{(c)}, \gamma^{(c)}, \bar{\gamma}^{(c)}, \underline{\gamma}^{(c)}\right) \\
& \left(\beta^{\infty}, \bar{\beta}^{\infty}, \underline{\beta}^{\infty}, \beta^{\prime \infty}, \bar{\beta}^{\prime \infty}, \underline{\beta}^{\prime \infty}, \gamma^{\infty}, \bar{\gamma}^{\infty}, \underline{\gamma}^{\infty}, \gamma^{\prime \infty}, \bar{\gamma}^{\infty}, \underline{\gamma}^{\prime \infty}\right) .
\end{aligned}
$$

Alors, les douze processus limites sont des mouvements browniens réels issus de 0 , dont la loi conjointe vérifie les cinq propriétés suivantes

(a) $\bar{\beta}^{\infty}$, respectivement $\underline{\beta}^{\infty}, \bar{\gamma}^{\infty}, \underline{\gamma}^{\infty}$, est le mouvement brownien associé à la martingale $\int_{0}^{\cdot} d \beta_{s}^{\infty} I\left(\beta_{s}^{\infty} \geq a \hat{\beta}_{s}^{\infty}\right)$, respectivement $\int_{0}^{\cdot} d \beta_{s}^{\infty} I\left(\beta_{s}^{\infty}<a \hat{\beta}_{s}^{\infty}\right)$, $\int_{0}^{\cdot} d \gamma_{s}^{\infty} I\left(\beta_{s}^{\infty} \geq a \hat{\beta}_{s}^{\infty}\right), \int_{0}^{\cdot} d \gamma_{s}^{\infty} I\left(\beta_{s}^{\infty}<a \hat{\beta}_{s}^{\infty}\right)$, et de même pour $\bar{\beta}^{\infty}, \underline{\beta}^{\prime \infty}$, $\bar{\gamma}^{\infty}, \underline{\gamma}^{\prime \infty}$

(b) $\overline{\boldsymbol{\beta}}^{\infty}=\overline{\boldsymbol{\beta}}^{\infty}$ et $\bar{\gamma}^{\infty}=\bar{\gamma}^{\prime \infty}$;

(c) les six mouvements browniens $\bar{\beta}^{\infty}, \underline{\beta}^{\infty}, \underline{\beta}^{\infty}, \bar{\gamma}^{\infty}, \underline{\gamma}^{\infty}, \underline{\gamma}^{\prime \infty}$ sont indépendants;

(d) $\underline{\beta}^{\infty}$ et $\beta^{\prime \infty}$ sont indépendants, et $\underline{\beta}^{\prime \infty}$ et $\beta^{\infty}$ sont indépendants;

(e) pour tout $\varepsilon>0$, si $T_{\varepsilon}\left(\beta^{\infty}\right)=\inf \left\{t ; \beta_{t}^{\infty}=\varepsilon\right\}$, resp. $T_{\varepsilon}\left(\beta^{\prime \infty}\right)=$ $\inf \left\{t ; \beta_{t}^{\prime \infty}=\varepsilon\right\}$, on $a$

$$
\int_{0}^{T_{e}\left(\beta^{\infty}\right)} d s I\left(\beta_{s}^{\infty} \geq a \hat{\beta}_{s}^{\infty}\right)=\int_{0}^{T_{e}\left(\beta^{\prime \infty}\right)} d s I\left(\beta_{s}^{\prime \infty} \geq a \hat{\beta}_{s}^{\prime \infty}\right) .
$$

Remarques. (i) On peut montrer que les propriétés (a), ., (e) caractérisent la loi conjointe des douze processus $\beta^{\infty}, \ldots, \underline{\gamma}^{\prime \infty}$, ce qui, grâce à un argument simple de tension permet d'affirmer que la convergence du Lemme 5.3 a effectivement lieu quand $c$ croît vers $+\infty$ (pas seulement le long d'une suite $\left(c_{n}\right)$ ). Comme nous n'utiliserons pas ce résultat, nous en laissons la démonstration au lecteur.

(ii) Il semble plausible que les propriétés $(a),(b),(c)$ suffisent à caractériser la loi limite du lemme (cela est vrai, et facile à vérifier, lorsque $a=0$ ), et que 
les propriétés (d) et (e) en soient alors des conséquences. Pour s'en assurer, il suffirait de montrer que l'énoncé du Lemme 5.2 reste vrai pour un mouvement brownien réel issu de 0 (au lieu de $\varepsilon>0$ ). On peut voir que c'est le cas au moins lorsque $a>-1$ (voir [14] et la discussion de la fin de la partie 6 du présent travail). Dans la suite, nous proposons des démonstrations qui s'appliquent au cas général $a \in$ ] $-\infty ; 1$ [, mais nous attirons l'attention du lecteur sur le fait que ces démonstrations pourraient être sensiblement simplifiées pour $a>-1$.

Démonstration du Théorème 5.1. Nous admettons provisoirement les résultats des Lemmes 5.2 et 5.3. Nous avons vu dans la démonstration du Théorème 4.2 que:

$$
\frac{2}{\log t} \theta_{t}-\gamma^{(c)}\left(T\left(\beta^{(c)}\right)\right) \underset{t \rightarrow \infty}{\stackrel{(P)}{\rightarrow}} 0
$$

où l'on note toujours $c=\frac{1}{2} \log t$. On a aussi le résultat analogue pour $\theta_{t}^{\prime}$. Soit maintenant $\left(c_{n}\right)$ une suite croissant vers $+\infty$ telle que la convergence du Lemme 5.3 ait lieu le long de $\left(c_{n}\right)$. Un argument évident de tension montre que, de toute suite croissant vers $+\infty$, on peut extraire une sous-suite ayant cette propriété. En notant $t_{n}=\exp \left(2 c_{n}\right)$, on a, avec les notations du Lemme 5.3 ,

$$
\frac{2}{\log t_{n}}\left(\theta_{t_{n}}, \theta_{t_{n}}^{\prime}\right) \underset{n \rightarrow \infty}{\stackrel{(d)}{\rightarrow}}\left(\gamma^{\infty}\left(T\left(\beta^{\infty}\right)\right), \gamma^{\prime \infty}\left(T\left(\beta^{\prime \infty}\right)\right)\right) .
$$

Grâce à nouveau à un argument de tension, il suffit pour compléter la démonstration du Théorème 5.1 de voir que la loi limite de (5.e) coïncide avec celle de l'énoncé du Théorème 5.1. Pour cela, nous utiliserons les propriétés $(\mathrm{a}), \ldots,(\mathrm{e})$ du Lemme 5.3, dont nous allons voir qu'elles suffisent à caractériser la loi de $\left(\gamma^{\infty}\left(T\left(\beta^{\infty}\right)\right), \gamma^{\prime \infty}\left(T\left(\beta^{\prime \infty}\right)\right)\right)$ (il n'est pas nécessaire de savoir qu'elles carac térisent la loi de $\left(\beta^{\infty}, \beta^{\prime \infty}, \gamma^{\infty}, \gamma^{\prime \infty}\right)$; voir cependant les remarques ci-dessus).

On observe d'abord que:

$$
\begin{aligned}
\gamma^{\infty}\left(T\left(\beta^{\infty}\right)\right)= & \bar{\gamma}^{\infty}\left(\int_{0}^{T\left(\beta^{\infty}\right)} d s I\left(\beta_{s}^{\infty} \geq a \hat{\beta}_{s}^{\infty}\right)\right) \\
& +\underline{\gamma}^{\infty}\left(\int_{0}^{T\left(\beta^{\infty}\right)} d s I\left(\beta_{s}^{\infty}<a \hat{\beta}_{s}^{\infty}\right)\right), \\
\gamma^{\prime \infty}\left(T\left(\beta^{\infty}\right)\right)= & \bar{\gamma}^{\infty}\left(\int_{0}^{T\left(\beta^{\prime \infty}\right)} d s I\left(\beta_{s}^{\prime \infty} \geq a \hat{\beta}_{s}^{\prime \infty}\right)\right) \\
& +\underline{\gamma}^{\prime \infty}\left(\int_{0}^{T\left(\beta^{\prime \infty}\right)} d s I\left(\beta_{s}^{\prime \infty}<a \hat{\beta}_{s}^{\prime \infty}\right)\right)
\end{aligned}
$$

et on utilise les remarques suivantes.

(i) D'après le Lemme 5.3 , les trois mouvements browniens $\omega_{1}:=\underline{\gamma}^{\infty}$, $\omega_{1}^{\prime}:=\underline{\gamma}^{\prime \infty}$ et $\omega_{2}:=\bar{\gamma}^{\infty}=\bar{\gamma}^{\infty}$ sont indépendants et indépendants du couple $\left(\beta^{\infty}, \beta^{\prime \infty}\right)$. 
(ii) Soit $\sigma_{2}=\int_{0}^{T\left(\beta^{\infty}\right)} d s I\left(\beta_{s}^{\infty} \geq a \hat{\beta}_{s}^{\infty}\right)$. On a: $\sigma_{2}=\lim _{\varepsilon \rightarrow 0} \sigma_{2}^{\varepsilon}$, où, avec les notations du Lemme 5.3(e),

$$
\sigma_{2}^{\varepsilon}=\int_{T_{\varepsilon}\left(\beta^{\infty}\right)}^{T\left(\beta^{\infty}\right)} d s I\left(\beta_{s}^{\infty} \geq a \hat{\beta}_{s}^{\infty}\right)
$$

Pour tout $\varepsilon \in] 0 ; 1[$, posons

$$
\eta_{t}^{\varepsilon}=\beta_{T_{\varepsilon}\left(\beta^{\infty}\right)+t}^{\infty}, \quad \eta_{t}^{\ell}=\beta_{T_{\varepsilon}\left(\beta^{\prime \infty}\right)+t}^{\prime \infty} \quad(t \geq 0) .
$$

Les processus $\eta^{\varepsilon}, \eta^{\prime}$ sont deux mouvements browniens réels issus de $\varepsilon$. Avec les notations du Lemme 5.2, on a: $\bar{\eta}^{\varepsilon}=\bar{\eta}^{\ell}$. En effet, on voit aisément que pour tout $t \geq 0$,

$$
\begin{aligned}
& \bar{\eta}_{t}^{\varepsilon}=\bar{\beta}^{\infty}\left(\int_{0}^{T_{\varepsilon}\left(\beta^{\infty}\right)} d s I\left(\beta_{s}^{\infty} \geq a \hat{\beta}_{s}^{\infty}\right)+t\right), \\
& \bar{\eta}_{t}^{\varepsilon}=\bar{\beta}^{\prime \infty}\left(\int_{0}^{T_{e}\left(\beta^{\prime \infty}\right)} d s I\left(\beta_{s}^{\prime \infty} \geq a \hat{\beta}_{s}^{\prime \infty}\right)+t\right)
\end{aligned}
$$

d'où le résultat recherché grâce aux propriétés (b) et (e) du Lemme 5.3.

On observe ensuite que

$$
\sigma_{2}^{\varepsilon}=\int_{0}^{T\left(\eta^{\varepsilon}\right)} d s I\left(\eta_{s}^{\varepsilon} \geq a \hat{\eta}_{s}^{\varepsilon}\right),
$$

et le Lemme 5.2(ii) montre que $\sigma_{2}^{\varepsilon}$ est une fonctionnelle de $\bar{\eta}^{\varepsilon}$. L'égalité $\bar{\eta}^{\varepsilon}=\bar{\eta}^{\varepsilon}$ entraîne donc avec des notations évidentes que $\sigma_{2}^{\varepsilon}=\sigma_{2}^{\prime \varepsilon}$, d'où en faisant tendre $\varepsilon$ vers 0 ,

$$
\sigma_{2}=\sigma_{2}^{\prime}=\int_{0}^{T\left(\beta^{\prime \infty}\right)} d s I\left(\beta_{s}^{\prime \infty} \geq a \hat{\beta}_{s}^{\prime \infty}\right) .
$$

(iii) Soit $\left(l_{t}^{(a)}, t \geq 0\right)$ le temps local en 0 du processus $\left(\beta_{t}^{\infty}-a \hat{\beta}_{t}^{\infty}, t \geq 0\right)$. On a

$$
l_{T\left(\beta^{\infty}\right)}^{(a)}=\lim _{\varepsilon \rightarrow 0}\left(l_{T\left(\beta^{\infty}\right)}^{(a)}-l_{T_{\varepsilon}\left(\beta^{\infty}\right)}^{(a)}\right) .
$$

Les mêmes arguments qu'en (ii) ci-dessus, utilisant à nouveau le Lemme 5.2, montrent que

$$
l_{T\left(\beta^{\infty}\right)}^{(a)}=l_{T\left(\beta^{\prime \infty}\right)}^{(a)}=: l^{(a)} .
$$

(iv) Le couple $\left(\sigma_{2}, l^{(a)}\right)$ est indépendant de la tribu $\sigma\left(\underline{\beta}^{\infty}, \underline{\beta}^{\prime \infty}\right)$ engendrée par les processus $\underline{\beta}^{\infty}$ et $\underline{\beta}^{\prime \infty}$. Pour le voir, on écrit, avec les notations de (ii) et (iii) ci-dessus,

$$
\left(\sigma_{2}, l^{(a)}\right)=\lim _{\varepsilon \rightarrow 0}\left(\sigma_{2}^{\varepsilon}, l_{T\left(\beta^{\infty}\right)}^{(a)}-l_{T_{\varepsilon}\left(\beta^{\infty}\right)}^{(a)}\right)
$$

et, puisque pour tout $\varepsilon>0$, les variables $\sigma_{2}^{\varepsilon}$ et $l_{T\left(\beta^{\infty}\right)}^{(a)}-l_{T_{\varepsilon}\left(\beta^{\infty}\right)}^{(a)}$ sont mesurables par rapport à $\sigma\left(\bar{\eta}^{\varepsilon}\right)$, il suffit d'établir que $\bar{\eta}^{\varepsilon}$ est indépendant de $\sigma\left(\underline{\beta}^{\infty}, \underline{\beta}^{\prime \infty}\right)$. 
Or, la propriété de Markov forte au temps $T_{\varepsilon}\left(\beta^{\infty}\right)$ puis le Lemme 5.2 entraînent que $\bar{\eta}^{\varepsilon}$ est indépendant de

$$
\sigma\left(\beta_{s \wedge T_{\varepsilon}\left(\beta^{\infty}\right)}^{\infty}, s \geq 0\right) \vee \sigma\left(\underline{\eta}^{\varepsilon}\right),
$$

tribu qui contient manifestement $\sigma\left(\underline{\beta}^{\infty}\right)$. D'après le Lemme 5.3(d), $\underline{\beta}^{\prime \infty}$ est indépendant de $\beta^{\infty}$ donc a fortiori de $\sigma\left(\bar{\eta}^{\varepsilon}, \underline{\beta}^{\infty}\right)$ et on conclut que $\bar{\eta}^{\varepsilon}$ est indépendant de $\sigma\left(\underline{\beta}^{\infty}, \underline{\beta}^{\prime \infty}\right)$.

(v) Pour terminer, on observe, soit en faisant tendre $\varepsilon$ vers 0 dans la formule (5.d) appliquée à $\eta^{\varepsilon}$, soit par une démonstration directe, que

$$
\begin{aligned}
& \sigma_{1}:=\int_{0}^{T\left(\beta^{\infty}\right)} d s I\left(\beta_{s}^{\infty}<a \hat{\beta}_{s}^{\infty}\right)=\inf \left\{t ; \underline{\beta}_{s}^{\infty}=\frac{1}{2} l^{(a)}\right\}, \\
& \sigma_{1}^{\prime}:=\int_{0}^{T\left(\beta^{\prime \infty}\right)} d s I\left(\beta_{s}^{\prime \infty}<a \hat{\beta}_{s}^{\prime \infty}\right)=\inf \left\{t ; \underline{\beta}_{s}^{\prime \infty}=\frac{1}{2} l^{(a)}\right\} .
\end{aligned}
$$

Compte-tenu des remarques précédentes, on voit que, conditionnellement à la variable $l^{(a)}$, les variables $\sigma_{1}$ et $\sigma_{1}^{\prime}$ sont indépendantes, et indépendantes de $\sigma_{2}$. De plus, leur loi conditionnelle est celle de $\frac{1}{4}\left(l^{(a)}\right)^{2} T_{1}$. Ceci termine la démonstration du Théorème 5.1, modulo la preuve de la formule (5.c), qui sera donnée dans la partie 6 .

Preuve du Lemme 5.3. Commençons par établir l'assertion (a). On remarque d'abord que $\bar{\beta}^{(c)}$ est aussi le mouvement brownien associé à $(1 / c) \bar{M}_{\text {. }}$, soit encore, après changement de temps, à

$$
\int_{0}^{\cdot} d \beta_{s}^{(c)} I\left(\left|W_{\tau_{c^{2} s}}-f\left(Z_{\tau_{c^{2} s}}\right)\right| \geq \tau_{c^{2} s}^{a / 2}\right)=\int_{0}^{\cdot} d \beta_{s}^{(c)} I\left(\frac{1}{c} \rho_{\tau_{c^{2} s}} \geq \frac{a}{2 c} \log \tau_{c^{2} s}\right) .
$$

Or, d'après la Proposition 4.3, pour tout $s>0$,

$$
\frac{1}{c} \rho_{\tau_{c^{2} s}}-\beta_{s}^{(c)} \underset{c \rightarrow \infty}{\stackrel{(P)}{\rightarrow}} 0 \quad \text { et } \quad \frac{1}{2 c} \log \left(\tau_{c^{2} s}\right)-\hat{\beta}_{s}^{(c)} \underset{c \rightarrow \infty}{\stackrel{(P)}{\rightarrow}} 0 .
$$

On en déduit que, pour tout $t>0$,

$$
\int_{0}^{t} d s I\left(\frac{1}{c} \rho_{\tau_{c^{2} s}} \geq \frac{a}{2 c} \log \tau_{c^{2} s}\right)-\int_{0}^{t} d s I\left(\beta_{s}^{(c)} \geq a \hat{\beta}_{s}^{(c)}\right) \underset{c \rightarrow \infty}{\stackrel{(P)}{\rightarrow}} 0
$$

d'où aussi

$$
\int_{0}^{t} d \beta_{s}^{(c)} I\left(\frac{1}{c} \rho_{\tau_{c^{2} s}} \geq \frac{a}{2 c} \log \tau_{c^{2} s}\right)-\int_{0}^{t} d \beta_{s}^{(c)} I\left(\beta_{s}^{(c)} \geq a \hat{\beta}_{s}^{(c)}\right) \underset{c \rightarrow \infty}{\stackrel{(P)}{\rightarrow}} 0 .
$$

Soient $\tilde{\beta}^{(c)}$, resp. $\tilde{\beta}^{\infty}$, le mouvement brownien associé à

$$
\int_{0}^{\cdot} d \beta_{s}^{(c)} I\left(\beta_{s}^{(c)} \geq a \hat{\beta}_{s}^{(c)}\right), \quad \text { resp. } \quad \int_{0}^{\cdot} d \beta_{s}^{\infty} I\left(\beta_{s}^{\infty} \geq a \hat{\beta}_{s}^{\infty}\right) .
$$

Il découle de (5.f) que, pour tout $t \geq 0$,

$$
\bar{\beta}_{t}^{(c)}-\tilde{\beta}_{t}^{(c)} \underset{c \rightarrow \infty}{\stackrel{(P)}{\rightarrow}} 0
$$


d'où $\bar{\beta}^{\infty}=\tilde{\beta}^{\infty}$, ce qui était le résultat recherché. On démontre de même les assertions similaires relatives à $\underline{\beta}^{\infty}, \bar{\gamma}^{\infty}$ et $\underline{\gamma}^{\infty}$.

Passons maintenant à la preuve de (b). Rappelons que $\bar{\beta}$, resp. $\bar{\beta}^{\prime}$, est le mouvement brownien associé à $\bar{M}$, resp. $\bar{M}^{\prime}$. Or, d'après la Proposition 4.3,

$$
\frac{1}{(\log t)^{2}}\langle\bar{M}\rangle_{t} \underset{t \rightarrow \infty}{\stackrel{(d)}{\rightarrow}} \frac{1}{4} \int_{0}^{T\left(\beta^{\infty}\right)} d s I\left(\beta_{s}^{\infty} \geq a \hat{\beta}_{s}^{\infty}\right)
$$

et de même pour $\left\langle\bar{M}^{\prime}\right\rangle_{t}$. Notons en particulier que la variable limite dans (5.g) est strictement positive p.s. En utilisant la remarque suivant le Théorème 2.3, on voit qu'il suffit, pour montrer l'égalité $\bar{\beta}^{\infty}=\bar{\beta}^{\prime \infty}$, d'établir que

$$
\frac{1}{(\log t)^{2}}\left\langle\bar{M}-\bar{M}^{\prime}\right\rangle_{t} \underset{t \rightarrow \infty}{\stackrel{(P)}{\rightarrow}} 0
$$

soit encore

$$
\begin{aligned}
\frac{1}{(\log t)^{2}} \int_{0}^{t} d s \mid & \frac{1}{W_{s}-f\left(Z_{s}\right)} I\left(\left|W_{s}-f\left(Z_{s}\right)\right| \geq s^{a / 2}\right) \\
& -\left.\frac{1}{W_{s}-g\left(Z_{s}\right)} I\left(\left|W_{s}-g\left(Z_{s}\right)\right| \geq s^{a / 2}\right)\right|^{2} \underset{t \rightarrow \infty}{\stackrel{(P)}{\rightarrow} 0 .}
\end{aligned}
$$

En utilisant l'hypothèse (5.a), la loi du logarithme itéré pour le processus $Z$, et la Proposition 4.3(ii), on se ramène à montrer que pour tout $\varepsilon>0$,

$$
\frac{1}{(\log t)^{2}} \int_{0}^{i} d s\left|\frac{1}{W_{s}-f\left(Z_{s}\right)}-\frac{1}{W_{s}-g\left(Z_{s}\right)}\right|^{2} I\left(\left|W_{s}-f\left(Z_{s}\right)\right| \geq s^{(a / 2)+\varepsilon}\right) \underset{t \rightarrow \infty}{\stackrel{(P)}{\rightarrow}} 0 .
$$

Or, la preuve de cette dernière assertion est facile: on utilise à nouveau l'hypothèse (5.a) et la loi du logarithme itéré pour $Z$, qui montrent que, pour tout $\delta>0$, on a, pour tout $s$ assez grand tel que $\left|W_{s}-f\left(Z_{s}\right)\right| \geq s^{(a / 2)+\varepsilon}$,

$$
\left|\frac{1}{W_{s}-f\left(Z_{s}\right)}-\frac{1}{W_{s}-g\left(Z_{s}\right)}\right| \leq \delta \cdot \frac{1}{\left|W_{s}-f\left(Z_{s}\right)\right|}
$$

(noter que pour $s$ assez grand la condition $\left|W_{s}-f\left(Z_{s}\right)\right| \geq s^{(a / 2)+\varepsilon}$ entraîne que $\left|f\left(Z_{s}\right)-g\left(Z_{s}\right)\right|$ est petit devant $\left.\left|W_{s}-f\left(Z_{s}\right)\right|\right)$. On conclut en choisissant $\delta$ arbitrairement petit et en appliquant la Proposition 4.3(ii). On montre de même que $\bar{\gamma}^{\infty}=\bar{\gamma}^{\infty}$.

Montrons maintenant la propriété (c). On sait que $\underline{\beta}$, resp. $\underline{\beta^{\prime}}, \bar{\beta}, \underline{\gamma}$, $\underline{\gamma}^{\prime}, \bar{\gamma}$, est le mouvement brownien associé à $\underline{M}$, resp. $\underline{M^{\prime}}, \bar{M}, \underline{N}, \underline{N^{\prime}}, \overline{\bar{N}}$. Si $R$ désigne l'une des six martingales locales $\underline{M}, \underline{M^{\prime}}, \bar{M}, \underline{N}, \underline{N}^{\prime}, \bar{N}$, la Proposition 4.3 montre que

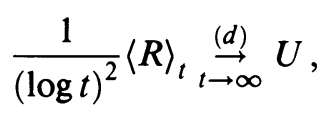

où $U$ est une variable aléatoire p.s. strictement positive. Compte-tenu du Théorème 2.2, il suffit pour établir la propriété (c) de montrer que, si $R$ et 
$S$ sont deux martingales parmi les six mentionnées plus haut, on a

$$
\frac{1}{(\log t)^{2}} \int_{0}^{t}\left|d\langle R, S\rangle_{s}\right| \stackrel{(P)}{\stackrel{(P)}{\rightarrow}} 0
$$

Cela nous donne en principe quinze vérifications à faire. Sur ces quinze vérifications, sept sont immédiates, puisque les crochets $\langle\underline{M}, \bar{M}\rangle,\langle\underline{M}, \underline{N}\rangle,\langle\underline{M}, \bar{N}\rangle$, $\left\langle\underline{M}^{\prime}, \underline{N}^{\prime}\right\rangle,\langle\bar{M}, \underline{N}\rangle,\langle\bar{M}, \bar{N}\rangle,\langle\underline{N}, \bar{N}\rangle$ sont, par construction, identiquement nuls. Les quatre vérifications concernant $\left\langle\underline{M}^{\prime}, \bar{M}\right\rangle,\left\langle\underline{M^{\prime}}, \bar{N}\right\rangle,\left\langle\underline{N}^{\prime}, \bar{M}\right\rangle$, $\left\langle\underline{N}^{\prime}, \bar{N}\right\rangle$ sont faciles, compte-tenu de la preuve de (b): on écrit, par exemple pour la première,

$$
\int_{0}^{t}\left|d\left\langle\underline{M^{\prime}}, \bar{M}\right\rangle_{s}\right| \leq \int_{0}^{t}\left|d\left\langle\underline{M}^{\prime}, \bar{M}^{\prime}\right\rangle_{s}\right|+\int_{0}^{t}\left|d\left\langle\underline{M}^{\prime}, \bar{M}-\bar{M}^{\prime}\right\rangle_{s}\right|
$$

et d'une part $\left\langle\underline{M}^{\prime}, \bar{M}^{\prime}\right\rangle \equiv 0$, d'autre part

$$
\int_{0}^{t}\left|d\left\langle\underline{M^{\prime}}, \bar{M}-\bar{M}^{\prime}\right\rangle_{s}\right| \leq\left\langle\underline{M^{\prime}}\right\rangle_{t}^{1 / 2}\left\langle\bar{M}-\bar{M}^{\prime}\right\rangle_{t}^{1 / 2}
$$

et on a vu que

$$
\frac{1}{(\log t)^{2}}\left\langle\bar{M}-\bar{M}^{\prime}\right\rangle \underset{t}{\stackrel{(P)}{\longrightarrow}} 0
$$

et que $\left(1 /(\log t)^{2}\right)\left\langle\underline{M}^{\prime}\right\rangle_{t}$ est borné en probabilité.

Il reste à faire les quatre vérifications concernant $\left\langle\underline{M}, \underline{M}^{\prime}\right\rangle,\left\langle\underline{M}, \underline{N^{\prime}}\right\rangle$, $\left\langle\underline{M}^{\prime}, \underline{N}\right\rangle,\left\langle\underline{N^{\prime}}, \underline{N}\right\rangle$. Nous traiterons en détail le cas de la première, les autres preuves étant très semblables. On a

$$
\begin{aligned}
& \int_{0}^{t}\left|d\left\langle\underline{M}, \underline{M}^{\prime}\right\rangle_{s}\right|=\int_{0}^{t} d s\left|\frac{1}{W_{s}-f\left(Z_{s}\right)} \cdot \frac{1}{W_{s}-g\left(Z_{s}\right)}\right| \\
& \times I\left(\left|W_{s}-f\left(Z_{s}\right)\right|<s^{a / 2} ;\left|W_{s}-g\left(Z_{s}\right)\right|<s^{a / 2}\right)
\end{aligned}
$$

où $a \cdot b$ désigne le produit scalaire euclidien de $a$ et $b$. Comme dans la preuve de (b), on peut utiliser la Proposition 4.3(ii) et se ramener à montrer, pour tout $\varepsilon>0$,

$$
\begin{aligned}
\frac{1}{(\log t)^{2}} \int_{0}^{t} d s \mid \frac{1}{W_{s}-} & f\left(Z_{s}\right) \\
\quad & \frac{1}{W_{s}-g\left(Z_{s}\right)} \mid \\
& \quad I\left(\left|W_{s}-f\left(Z_{s}\right)\right| \leq s^{a / 2-\varepsilon} ;\left|W_{s}-g\left(Z_{s}\right)\right| \leq s^{a / 2-\varepsilon}\right) \underset{t \rightarrow \infty}{\stackrel{(P)}{\rightarrow}} 0 .
\end{aligned}
$$

Nous établirons cette dernière assertion en montrant que, pour $s$ assez grand, on ne peut pas avoir simultanément $\left|W_{s}-g\left(Z_{s}\right)\right|<s^{a / 2-\varepsilon}$ et $\left|W_{s}-g\left(Z_{s}\right)\right|<s^{a / 2-\varepsilon}$. Pour cela choisissons $\delta, \delta^{\prime}>0$ tels que $|a| \delta+\delta^{\prime}<\varepsilon$. En utilisant la loi du logarithme itéré pour $Z$, et le Corollaire 2.5 , on trouve que, pour $s$ grand, la condition $\left|W_{s}-g\left(Z_{s}\right)\right| \leq s^{a / 2-\varepsilon}$ entraîne

$$
s^{1 / 2-\delta} \leq\left|Z_{s}\right| \leq s^{1 / 2+\delta},
$$


d'où aussi, toujours pour $s$ grand,

$$
\left|(f-g)\left(Z_{s}\right)\right| \geq s^{a / 2-|a| \delta-\delta^{\prime}}>2 s^{a / 2-\varepsilon}
$$

et donc:

$$
\left|W_{s}-g\left(Z_{s}\right)\right| \geq\left|(f-g)\left(Z_{s}\right)\right|-\left|W_{s}-f\left(Z_{s}\right)\right|>s^{a / 2-\varepsilon},
$$

ce qui est le résultat recherché.

La preuve de (d) est immédiate compte-tenu des estimations qui précèdent. On écrit:

$$
\frac{1}{(\log t)^{2}}\left\langle M, \underline{M}^{\prime}\right\rangle_{t}=\frac{1}{(\log t)^{2}}\left(\left\langle\bar{M}, \underline{M}^{\prime}\right\rangle_{t}+\left\langle\underline{M}, \underline{M^{\prime}}\right\rangle_{t}\right) \underset{t \rightarrow \infty}{\stackrel{(P)}{\rightarrow}} 0
$$

d'après les estimations ci-dessus. L'indépendance de $\beta^{\infty}$ et $\underline{\beta}^{\infty}$ découle ensuite du Théorème 2.2.

Il reste à montrer (e). Ceci ne présente pas non plus de difficulté: une extension facile de la Proposition 4.3(ii) montre que

$$
\begin{gathered}
\int_{0}^{T_{\ell}\left(\beta^{\infty}\right)} d s I\left(\beta_{s}^{\infty} \geq a \hat{\beta}_{s}^{\infty}\right)-\int_{0}^{T_{\ell}\left(\beta^{\prime \infty}\right)} d s I\left(\beta_{s}^{\prime \infty} \geq a \hat{\beta}_{s}^{\prime \infty}\right) \\
=\lim _{t \rightarrow \infty} \frac{4}{(\log t)^{2}}\left(\langle\bar{M}\rangle_{t^{\varepsilon}}-\left\langle\bar{M}^{\prime}\right\rangle_{t^{\varepsilon}}\right)=0,
\end{gathered}
$$

puisque nous avons remarqué plus haut que

$$
\lim _{t \rightarrow \infty} \frac{4}{(\log t)^{2}}\left(\left\langle\bar{M}-\bar{M}^{\prime}\right\rangle_{t}\right)=0 .
$$

\section{A PROPOS DE CERTAINES FONCTIONNELLES DU MOUVEMENT BROWNIEN LINEAIRE}

(6.1) Nous nous proposons dans cette partie d'abord de calculer la transformée de Fourier-Laplace de la loi limite du Théorème 5.1, et ensuite de démontrer le Lemme 5.2.

Nous considérons un mouvement brownien réel $\eta=\left(\eta_{t}, t \geq 0\right)$ issu de 0 et, comme ci-dessus, nous notons $\hat{\eta}_{t}=\sup \left\{\eta_{s}, s \leq t\right\}, T(\eta)=\inf \left\{t ; \eta_{t}=1\right\}$, et $l_{T(\eta)}^{(a)}$ le temps local en 0 , au temps $T(\eta)$, de $\eta-a \hat{\eta}$.

Proposition 6.1. Soient $a \in]-\infty, 1\left[\right.$ et $\alpha=1-a$. Alors, pour tous $\lambda, \lambda^{\prime}$, $\lambda^{\prime \prime} \geq 0$,

$$
\begin{aligned}
E\left[\operatorname { e x p } \left(-\lambda \int_{0}^{T(\eta)} I\left(\eta_{s} \geq\right.\right.\right. & \left.\left.\left.a \hat{\eta}_{s}\right) d s-\lambda^{\prime} l_{T(\eta)}^{(a)}-\lambda^{\prime \prime} \int_{0}^{T(\eta)} I\left(\eta_{s}<a \hat{\eta}_{s}\right) d s\right)\right] \\
& =\left(\operatorname{ch} \alpha \sqrt{2 \lambda}+\frac{2 \lambda^{\prime}+\sqrt{2 \lambda^{\prime \prime}}}{\sqrt{2 \lambda}} \operatorname{sh} \alpha \sqrt{2 \lambda}\right)^{-1 / \alpha}
\end{aligned}
$$


Preuve. Soient $\beta$ un mouvement brownien réfléchi issu de 0 et $l=\left(l_{t}, t \geq 0\right)$ le temps local symétrique de $\beta$ en 0 . Soit $\tau=\inf \left\{t ; l_{t}>1\right\}$. L'identité de Lévy

$$
(\hat{\eta}-\eta, \hat{\eta}) \stackrel{(d)}{=}(\beta, l)
$$

montre que

$$
\left(\int_{0}^{T(\eta)} I\left(\eta_{s} \geq a \hat{\eta}_{s}\right) d s, l_{T(\eta)}^{(a)}, \int_{0}^{T(\eta)} I\left(\eta_{s}<a \hat{\eta}_{s}\right) d s\right)
$$

$$
\left(\int_{0}^{\tau} I\left(\beta_{s} \leq \alpha l_{s}\right) d s, L_{\tau}^{0}(\beta-\alpha l), \int_{0}^{\tau} I\left(\beta_{s}>\alpha l_{s}\right) d s\right)
$$

où $L_{\tau}^{0}(\beta-\alpha l)$ désigne le temps local en 0 , à l'instant $\tau$, de $\beta-\alpha l$.

Pour tout $s \geq 0$, soit $\tau_{s}=\inf \left\{t ; l_{t}>s\right\}$. Soit $C_{0}$ l'espace des fonctions continues à support compact de $\mathbf{R}_{+}$dans $\mathbf{R}_{+}$, auquel on adjoint un point cimetière $\partial$. Le processus $\left(e_{s}, s \geq 0\right)$ des excursions de $\beta$ est le processus à valeurs dans $C_{0}$ défini par

$$
\begin{aligned}
e_{s} & =\partial \\
e_{s}(u) & =\left\{\begin{array}{lll}
\beta_{\tau_{s^{-}}+u} & \left(u \leq \tau_{s}-\tau_{s^{-}}\right) & \text {si } \tau_{s^{-}}=\tau_{s}, \\
0 & \left(u>\tau_{s}\right) & \text { si } \tau_{s^{-}}<\tau_{s} .
\end{array}\right.
\end{aligned}
$$

D'après un célèbre théorème d'Itô [6], le processus $\left(e_{s}, s \geq 0\right)$ est un processus de Poisson ponctuel, dont nous notons $n(d e)$ la mesure caractéristique. On a, pour toute fonction $\varphi$ mesurable positive définie sur $\mathbf{R}_{+} \times C_{0}$,

$$
E\left[\exp -\sum_{s \geq 0} \varphi\left(s, e_{s}\right)\right]=\exp -\int_{0}^{\infty} d s \int n(d e)\left(1-e^{-\varphi(s, e)}\right) .
$$

On peut utiliser la formule (6.b) pour établir les deux formules bien connues suivantes (voir, par exemple, D. Williams [21, p. 99]): si

$$
\sigma(e)=\sup \{t ; e(t)>0\},
$$

pour tous $x>0, \lambda \geq 0$,

$$
\begin{gathered}
n\left(\sup _{t \geq 0} e(t) \geq x\right)=\frac{1}{x} \\
\int n(d e)\left(1-e^{-\lambda \sigma(e)}\right) I\left(\sup _{t \geq 0} e(t)<x\right)=\frac{1}{x}(x \sqrt{2 \lambda} \operatorname{coth} x \sqrt{2 \lambda}-1) .
\end{gathered}
$$


Nous pouvons maintenant établir le résultat de la proposition. Grâce à (6.a), on se ramène à étudier

$$
\text { (6.e) } \begin{array}{r}
E\left[\exp \left(-\lambda \int_{0}^{\tau} I\left(\beta_{s} \leq \alpha l_{s}\right) d s-\lambda^{\prime} L_{\tau}^{0}(\beta-\alpha l)-\lambda^{\prime \prime} \int_{0}^{\tau} I\left(\beta_{s}>\alpha l_{s}\right) d s\right)\right] \\
=E\left[\exp -\sum_{s \leq 1}\left(\lambda \int_{0}^{\sigma\left(e_{s}\right)} I\left(e_{s}(u) \leq \alpha s\right) d u+\lambda^{\prime} L^{\alpha s}\left(e_{s}\right)\right.\right. \\
\left.\left.+\lambda^{\prime \prime} \int_{0}^{\sigma\left(e_{s}\right)} I\left(e_{s}(u)>\alpha s\right) d u\right)\right] \\
=\exp -\int_{0}^{1} d s \int n(d e)\left(1-\exp \left(-\lambda \int_{0}^{\sigma(e)} I(e(u) \leq \alpha s) d u\right.\right. \\
\left.\left.-\lambda^{\prime} L^{\alpha s}(e)-\lambda^{\prime \prime} \int_{0}^{\sigma(e)} I(e(u)>\alpha s) d u\right)\right)
\end{array}
$$

d'après (6.b). Nous avons noté $L^{x}(e)$ le temps local (à l'instant $\sigma(e)$ ) au niveau $x$ de l'excursion $e$.

On doit donc calculer, pour tout $x>0$,

$$
\begin{aligned}
\int n(d e)\left(1-\exp \left(-\lambda \int_{0}^{\sigma(e)} I(e(u) \leq x) d u\right.\right. & -\lambda^{\prime} L^{x}(e) \\
& \left.\left.-\lambda^{\prime \prime} \int_{0}^{\sigma(e)} I(e(u)>x) d u\right)\right) .
\end{aligned}
$$

Sur l'ensemble $\{\sup e(t)<x\}$, on a $L^{x}(e)=0$ et $\int_{0}^{\sigma(e)} I(e(u)>x) d u=0$, et on est donc ramené à la formule (6.d). Il reste à étudier l'intégrale sur l'ensemble $\{\sup e(t) \geq x\}$. A partir de maintenant, on se place sur cet ensemble et on pose

$$
\sigma_{1}(e)=\inf \{t ; e(t)=x\}, \quad \sigma_{2}(e)=\sup \{t ; e(t)=x\} .
$$

Sous la mesure $n$ conditionnée par $\{\sup e(t) \geq x\}$, les processus $(e(t), 0 \leq t \leq$ $\left.\sigma_{1}(e)\right)$ et $\left(e\left(\sigma_{1}(e)+t\right), 0 \leq t \leq \sigma(e)-\sigma_{1}(e)\right)$ sont indépendants; le premier suit la loi d'un processus de Bessel de dimension trois issu de 0 arrêté au premier instant, noté $T_{x}^{(3)}$, où il atteint $x$; le second suit la loi d'un mouvement brownien réel issu de $x$ arrêté quand il atteint 0 (voir par exemple [21]). En particulier, toujours sous la mesure $n$ conditionnée par $\{\sup e(t) \geq x\}$,

- $\sigma_{1}(e)$ et $\sigma(e)-\sigma_{2}(e)$ sont indépendantes, de même loi que $T_{x}^{(3)}$, et le couple $\left(\sigma_{1}(e), \sigma(e)-\sigma_{2}(e)\right)$ est indépendant du processus $\left(e\left(\sigma_{1}(e)+t\right), 0 \leq\right.$ $\left.t \leq \sigma_{2}(e)-\sigma_{1}(e)\right)$,

- la variable $L^{x}(e)$ suit une loi exponentielle de moyenne $2 x$,

- conditionnellement à $L^{x}(e)=y$, les variables

$$
\int_{\sigma_{1}(e)}^{\sigma_{2}(e)} I(e(t)>x) d t \quad \text { et } \quad \int_{\sigma_{1}(e)}^{\sigma_{2}(e)} I(e(t) \leq x) d t
$$


sont indépendantes, la première suit la loi de $\tau_{y / 2}$ et la seconde suit la loi conditionnelle de $\tau_{y / 2}$ sachant que $\sup _{s \leq \tau_{y / 2}} \beta_{s}<x$.

On sait que

$$
\begin{aligned}
& E\left[\exp -\lambda T_{x}^{(3)}\right]=x \sqrt{2 \lambda} / \operatorname{sh} x \sqrt{2 \lambda} \\
& E\left[\exp -\lambda \tau_{y}\right]=\exp -y \sqrt{2 \lambda}
\end{aligned}
$$

et il découle de (6.d) que

$$
E\left[\exp -\lambda \tau_{y} \mid \sup _{s \leq \tau_{y}} \beta_{s}<x\right]=\exp -\frac{y}{x}(x \sqrt{2 \lambda} \operatorname{coth} x \sqrt{2 \lambda}-1) .
$$

On peut maintenant utiliser (6.f), (6.g), (6.h) et les remarques précédentes pour obtenir

$$
\begin{aligned}
& \int_{\{\text {sup } e(t) \geq x\}} n(d e) \exp \left(-\lambda \int_{0}^{\sigma(e)} I(e(u) \leq x) d u-\lambda^{\prime} L^{x}(e)\right. \\
& \left.\quad-\lambda^{\prime \prime} \int_{0}^{\sigma(e)} I(e(u)>x) d u\right) \\
& =\frac{1}{x}\left(\frac{x \sqrt{2 \lambda}}{\operatorname{sh} x \sqrt{2 \lambda}}\right)^{2} \int_{0}^{\infty} \frac{d y}{2 x} e^{-y / 2 x} \exp -\left(\frac{y}{2 x}(x \sqrt{2 \lambda} \operatorname{coth} x \sqrt{2 \lambda}-1)\right. \\
& =\frac{\sqrt{2 \lambda}}{\operatorname{sh} x \sqrt{2 \lambda}} \cdot \frac{\left.-\lambda^{\prime} y-\frac{y}{2} \sqrt{2 \lambda^{\prime \prime}}\right)}{\sqrt{2 \lambda} \operatorname{ch} x \sqrt{2 \lambda}+\left(2 \lambda^{\prime}+\sqrt{2 \lambda^{\prime \prime}}\right) \operatorname{sh} x \sqrt{2 \lambda}} .
\end{aligned}
$$

En additionnant les intégrales sur les ensembles $\{\sup e(t)>x\}$ et $\{\sup e(t) \leq$ $x\}$, on trouve

$$
\begin{gathered}
\int n(d e)\left(1-\exp \left(-\lambda \int_{0}^{\sigma(e)} I(e(u) \leq x) d u-\lambda^{\prime} L^{x}(e)\right.\right. \\
\left.\left.-\lambda^{\prime \prime} \int_{0}^{\sigma(e)} I(e(u)>x) d u\right)\right) \\
=\frac{1}{x}(x \sqrt{2 \lambda} \operatorname{coth} x \sqrt{2 \lambda}-1) \\
\quad+\frac{1}{x}-\frac{\sqrt{2 \lambda}}{\operatorname{sh} x \sqrt{2 \lambda}} \frac{\sqrt{2 \lambda}}{\sqrt{2 \lambda} \operatorname{ch} x \sqrt{2 \lambda}+\left(2 \lambda^{\prime}+\sqrt{2 \lambda^{\prime \prime}}\right) \operatorname{sh} x \sqrt{2 \lambda}} \\
=\frac{2 \lambda \operatorname{sh} x \sqrt{2 \lambda}+\left(2 \lambda^{\prime}+\sqrt{2 \lambda^{\prime \prime}}\right) \sqrt{2 \lambda} \operatorname{ch} x \sqrt{2 \lambda}}{\sqrt{2 \lambda} \operatorname{ch} x \sqrt{2 \lambda}+\left(2 \lambda^{\prime}+\sqrt{2 \lambda^{\prime \prime}}\right) \operatorname{sh} x \sqrt{2 \lambda}} .
\end{gathered}
$$


Finalement, en utilisant (6.e),

$$
\begin{gathered}
E\left[\exp \left(-\lambda \int_{0}^{\tau} I\left(\beta_{s} \leq \alpha l_{s}\right) d s-\lambda^{\prime} L_{\tau}^{0}(\beta-\alpha l)-\lambda^{\prime \prime} \int_{0}^{\tau} I\left(\beta_{s}>\alpha l_{s}\right) d s\right)\right] \\
\quad=\exp -\int_{0}^{1} d u \frac{2 \lambda \operatorname{sh} \alpha u \sqrt{2 \lambda}+\left(2 \lambda^{\prime}+\sqrt{2 \lambda^{\prime \prime}}\right) \operatorname{ch} \alpha u \sqrt{2 \lambda}}{\sqrt{2 \lambda} \operatorname{ch} \alpha u \sqrt{2 \lambda}+\left(2 \lambda^{\prime}+\sqrt{2 \lambda^{\prime \prime}}\right) \operatorname{sh} \alpha u \sqrt{2 \lambda}} \\
=\left(\operatorname{ch} \alpha \sqrt{2 \lambda}+\frac{2 \lambda^{\prime}+\sqrt{2 \lambda^{\prime \prime}}}{\sqrt{2 \lambda}} \operatorname{sh} \alpha \sqrt{2 \lambda}\right)^{-1 / \alpha},
\end{gathered}
$$

d'où, compte-tenu de (6.a), le résultat recherché.

La formule (5.c) du Théorème 5.1 découle immédiatement de l'énoncé de la Proposition 6.1. En effet, avec les notations du Théorème 5.1,

$$
\begin{aligned}
& E\left[\exp i\left(\lambda_{1} \omega_{1}\left(\sigma_{1}\right)+\lambda_{1}^{\prime} \omega_{1}^{\prime}\left(\sigma_{1}^{\prime}\right)+\lambda_{2} \omega_{2}\left(\sigma_{2}\right)\right)\right] \\
& =E\left[\exp -\left(\frac{\lambda_{1}^{2}}{2} \sigma_{1}+\frac{\lambda_{1}^{\prime 2}}{2} \sigma_{1}^{\prime}+\frac{\lambda_{2}^{2}}{2} \sigma_{2}\right)\right] \\
& =E\left[\exp -\frac{1}{2}\left(\left(\frac{\lambda_{1}}{2} l_{T(\eta)}^{(a)}\right)^{2} T_{1}+\left(\frac{\lambda_{1}^{\prime}}{2} l_{T(\eta)}^{(a)}\right)^{2} T_{2}+\lambda_{2}^{2} \int_{0}^{T(\eta)} I\left(\eta_{s}>a \hat{\eta}_{s}\right) d s\right)\right] \\
& =E\left[\exp \left(-\frac{1}{2}\left(\lambda_{1}+\lambda_{1}^{\prime}\right) l_{T(\eta)}^{(a)}-\frac{\lambda_{2}^{2}}{2} \int_{0}^{T(\eta)} I\left(\eta_{s}>a \hat{\eta}_{s}\right) d s\right)\right]
\end{aligned}
$$

où nous avons d'abord utilisé l'identité (5.b) puis conditionné par rapport au processus $\eta$.

On pourrait donner d'autres démonstrations du résultat de la Proposition 6.1. Une possibilité consiste à utiliser les calculs de Knight [10] et Jeulin-Yor [8] qui fournissent des expressions explicites de

$$
E\left[\exp -\int_{0}^{\tau} d s q\left(\beta_{s}, l_{s}\right)\right],
$$

pour des fonctions $q$ de la forme

$q(x, l)=a_{1} I\left(0<x \leq k_{1}(l)\right)+\cdots+a_{n} I\left(k_{n-1}(l)<x \leq k_{n}(l)\right)+a_{n+1} I\left(k_{n}(l)<x\right)$.

On peut aussi utiliser l'invariance par changement d'échelle de la mesure d'Itô des excursions, qui, modulo quelques transformations simples, permet de déduire l'énoncé de la proposition du cas particulier $a=0$, lequel est bien connu (voir par exemple [18]). Un avantage de la démonstration ci-dessus est qu'elle explicite les transformées de Laplace, sous la mesure d'Itô $n$, de certaines fonctionnelles des excursions.

Pour tout réel $d \geq 0$, soit $C^{(d)}=\left(C_{y}^{(d)}, y \geq 0\right)$ le carré d'un processus de Bessel de dimension $d$ issu de 0 . La formule remarquable suivante:

$$
E\left[\exp \left(-\lambda \int_{0}^{x} C_{y}^{(d)} d y-\lambda^{\prime} C_{x}^{(d)}\right)\right]=\left(\operatorname{ch} x \sqrt{2 \lambda}+\frac{2 \lambda^{\prime}}{\sqrt{2 \lambda}} \operatorname{sh} x \sqrt{2 \lambda}\right)^{-d / 2},
$$


valable pour tous $\lambda, \lambda^{\prime} \geq 0, x>0$ (voir, par exemple, Pitman et Yor [17, p. 432]) peut être obtenue à partir du cas particulier $d=2$, en utilisant la propriété d'additivité des carrés de processus de Bessel.

En comparant cette formule avec la Proposition 6.1, et en utilisant (6.a), on trouve que

$$
\left(\int_{0}^{\tau} I\left(\beta_{s} \leq \alpha l_{s}\right) d s, L_{\tau}^{0}(\beta-\alpha l)\right) \stackrel{(d)}{=}\left(\int_{0}^{\alpha} C_{y}^{(2 / \alpha)} d y, C_{\alpha}^{(2 / \alpha)}\right) .
$$

Cette égalité en loi est expliquée, via la formule de densité de temps d'occupation, par l'identité plus générale: pour tout $x>0$,

$$
\left(L_{\tau}^{y-x}(\beta-x l), 0 \leq y \leq x\right) \stackrel{(d)}{=}\left(C_{y}^{(2 / x)}, 0 \leq y \leq x\right) .
$$

Cette dernière identité, qui est établie en [13], fournit la démonstration la plus directe de la Proposition 6.1.

(6.2) Le but de ce sous-paragraphe est de démontrer les différentes assertions du Lemme 5.2. Nous obtiendrons au passage un résultat d'unicité des solutions de certaines équations stochastiques, qui sera repris et précisé en [14]. Nous conservons les notations du Lemme 5.2; en particulier, $\eta=\left(\eta_{t}, t \geq 0\right)$ désigne un mouvement brownien réel issu de $\varepsilon$, pour un certain $\varepsilon \in] 0 ; 1[$. La formule de Tanaka montre que

$$
\left\{\begin{array}{l}
\left(\eta_{t}-a \hat{\eta}_{t}\right)_{+}=(1-a) \varepsilon+\int_{0}^{t} I\left(\eta_{s} \geq a \hat{\eta}_{s}\right) d \eta_{s}-a \hat{\eta}_{t}+\frac{1}{2} l_{t}^{(a)}, \\
\left(\eta_{t}-a \hat{\eta}_{t}\right)_{-}=-\int_{0}^{t} I\left(\eta_{s}<a \hat{\eta}_{s}\right) d \eta_{s}+\frac{1}{2} l_{t}^{(a)},
\end{array}\right.
$$

où $l_{t}^{(a)}$ désigne le temps local en 0 de $\eta-a \hat{\eta}$ à l'instant $t$. Posons pour tout $t \geq 0$,

$$
\begin{aligned}
& \tau_{t}^{+}=\inf \left\{s ; \int_{0}^{s} I\left(\eta_{u} \geq a \hat{\eta}_{u}\right) d u>t\right\}, \\
& \tau_{t}^{-}=\inf \left\{s ; \int_{0}^{s} I\left(\eta_{u}<a \hat{\eta}_{u}\right) d u>t\right\},
\end{aligned}
$$

et

Soit aussi

$$
U_{t}=\eta_{\tau_{t}^{+}}-a \hat{\eta}_{\tau_{t}^{+}}, \quad V_{t}=-\eta_{\tau_{t}^{-}}+a \hat{\eta}_{\tau_{t}^{-}}
$$

$$
\hat{U}_{t}=\sup _{s \leq t} U_{s}=\sup _{u \leq \tau_{t}^{+}}\left(\eta_{u}-a \hat{\eta}_{u}\right)_{+}=(1-a) \hat{\eta}_{\tau_{t}^{+}} .
$$

En remplaçant dans (6.i) $t$ par $\tau_{t}^{+}$, resp. par $\tau_{t}^{-}$, on trouve, compte-tenu de la définition de $\bar{\eta}$ et $\underline{\eta}$,

$$
\begin{gathered}
U_{t}=(1-a) \varepsilon+\bar{\eta}_{t}-\frac{a}{1-a} \hat{U}_{t}+L_{t}, \\
V_{t}=-\underline{\eta}_{t}+L_{t}^{\prime},
\end{gathered}
$$

où $L_{t}=\frac{1}{2} l_{\tau_{t}^{+}}^{(a)}$, resp. $L_{t}^{\prime}=\frac{1}{2} l_{\tau_{t}^{-}}^{(a)}$. On voit aisément que $L_{t}$, resp. $L_{t}^{\prime}$, est le temps local (symétrique) de $U^{t}$, resp. $V$, en 0 à l'instant $t$. 
L'équation (6.k) est l'équation de réflexion de Skorokhod dont la solution est donnée de manière explicite par:

$$
V_{t}=-\underline{\eta}_{t}+\sup _{s \leq t} \underline{\eta}_{s}
$$

En particulier, les filtrations naturelles de $V$ et $\underline{\eta}$ coïncident.

Considérons ensuite l'équation (6.j). Il découle de la Proposition 6.2 cidessous que $U$ est une fonctionnelle mesurable du mouvement brownien $\bar{\eta}$.

Nous pouvons maintenant démontrer les assertions du Lemme 5.2. Tout d'abord, le théorème de Knight sur les martingales continues orthogonales (Théorème 2.1 ci-dessus) montre que $\underline{\eta}$ et $\bar{\eta}$ sont indépendants. Ensuite, on voit immédiatement que

$$
\sigma_{2}=\inf \left\{t ; U_{t}=1-a\right\}
$$

et que $\frac{1}{2} l_{T(\eta)}^{(a)}$ coïncide avec le temps local en 0 de $U$ à l'instant $\sigma_{2}$ (le facteur $\frac{1}{2}$ vient de ce que nous considérons le temps local symétrique de $U$ en 0 ). On déduit de ces remarques que $\sigma_{2}$ et $l_{T(\eta)}^{(a)}$ sont mesurables par rapport à la tribu engendrée par $U$ donc aussi par rapport à $\mathscr{F}^{\bar{\eta}}$. D'autre part, on a

$$
\sigma_{2}=\inf \left\{t ; \tau_{t}^{-}>T(\eta)\right\}=\inf \left\{t ; L_{t}^{\prime}>\frac{1}{2} l_{T(\eta)}^{(a)}\right\}=\inf \left\{t ; \underline{\eta}_{t}>\frac{1}{2} l_{T(\eta)}^{(a)}\right\}
$$

puisque $L_{t}^{\prime}=\sup _{s \leq t} \underline{\eta}_{s}$ d'après (6.k) et (6.1).

Il reste à montrer que $\mathscr{F}^{\eta}=\mathscr{F}^{\bar{\eta}} \vee \mathscr{F}^{\eta}$, ce qui compte-tenu des remarques précédentes revient à prouver qu'on peut "reconstruire" $\eta$ à partir des deux processus réfléchis $U$ et $V$. La démonstration est la même que dans le cas particulier $a=0$, lequel est bien connu (voir par exemple [7, Lemme I-0-2]). Ceci termine la démonstration du Lemme 5.2, modulo la preuve de l'énoncé suivant:

Proposition 6.2. Soient $\varepsilon>0$ et $a \in \mathbf{R}$. Considérons l'équation stochastique:

$$
W_{t}=\varepsilon+B_{t}+a \hat{W}_{t}+L_{t}^{0}(W),
$$

où $B$ est un mouvement brownien linéaire issu de 0 , le processus inconnu $W$ est à valeurs positives, $\hat{W}_{t}=\sup \left\{W_{s}, s \leq t\right\}$ et $L_{t}^{0}(W)$ est le temps local symétrique de $W$ en 0 à l'instant $t$. Alors,

si $a \geq 1$, l'équation (6.m) n'a pas de solution;

si $a<1$, l'équation (6.m) a une unique solution, qui est adaptée à la filtration de $B$.

Preuve. Le cas $a \geq 1$ est trivial: en prenant $t=0$, on trouve $W_{0}>\hat{W}_{0}$ ce qui est absurde. Dans le cas $a<1$, on construit une suite $\left(T_{n}\right)$ de temps d'arrêt de la filtration de $B$ tels que $T_{n}$ croisse vers $\infty$, et, sur chaque intervalle stochastique $\left[0 ; T_{n}\right]$, l'équation (6.m) admette une unique solution qui s'écrit comme fonctionnelle explicite de $B$. On remarque d'abord que sur un petit intervalle $\left[0 ; \delta_{0}\right] \quad\left(\delta_{0}>0\right.$ aléatoire $)$ on doit avoir $W_{t}>0$ et donc sur cet intervalle

$$
W_{t}=\varepsilon+B_{t}+a \hat{W}_{t}
$$


ce qui conduit à l'expression explicite

$$
W_{t}=\frac{\varepsilon}{1-a}+B_{t}+\frac{a}{1-a} \sup _{s \leq t} B_{s} .
$$

Posons

$$
T_{1}=\inf \left\{t ; \frac{\varepsilon}{1-a}+B_{t}+\frac{a}{1-a} \sup _{s \leq t} B_{s}=0\right\} .
$$

Le raisonnement qui précède montre que, sur l'intervalle stochastique $\left[0 ; T_{1}\right]$, l'équation (6.m) a une unique solution donnée par (6.n).

$\mathrm{Si}$ on veut maintenant prolonger la solution, on remarque que, comme $W_{T_{1}}=$ $0, \widehat{W}$ ne croît pas sur un petit intervalle de la forme $\left[T_{1}, T_{1}+\delta_{1}\right]$, ce qui permet de se ramener à une équation de réflexion ordinaire. On trouve pour $t \in\left[T_{1}, T_{1}+\delta_{1}\right]:$

$$
W_{t}=B_{t}+\sup _{T_{1} \leq s \leq t}\left(-B_{s}\right) .
$$

On pose:

$$
T_{2}=\inf \left\{t>T_{1} ; B_{t}+\sup _{T_{1} \leq s \leq t}\left(-B_{s}\right)=\hat{W}_{T_{1}}\right\} .
$$

Sur l'intervalle stochastique $\left[0 ; T_{2}\right]$, l'équation $(6 . \mathrm{m})$ a une unique solution donnée par (6.n) sur $\left[0, T_{1}\right]$ et par (6.o) sur $\left[T_{1}, T_{2}\right]$.

On continue par récurrence la construction de la solution $W$ et de la suite $\left(T_{n}\right)$, de manière que sur chaque intervalle $\left[T_{2 n+1} ; T_{2 n+1}\right], W$ ne s'annule pas (et donc $L_{.}^{0}(W)$ ne croît pas) et sur chaque intervalle $\left[T_{2 n} ; T_{2 n+2}\right], \widehat{W}$ ne croît pas. Cela permet de résoudre l'équation $(6 . \mathrm{m})$ de proche en proche et montre aussi qu'il y a unicité de la solution. Par exemple, $T_{3}$ est défini par:

$$
T_{3}=\inf \left\{t>T_{2} ; \hat{W}_{T_{1}}+\left(B_{t}-B_{T_{2}}\right)+\frac{a}{1-a} \sup _{T_{2} \leq s \leq t}\left(B_{s}-B_{T_{2}}\right)=0\right\}
$$

et sur l'intervalle $\left[T_{2} ; T_{3}\right]$ on a

$$
W_{t}=\hat{W}_{T_{1}}+\left(B_{t}-B_{T_{2}}\right)+\frac{a}{1-a} \sup _{T_{2} \leq s \leq t}\left(B_{s}-B_{T_{2}}\right) .
$$

Puis $T_{4}$ est défini par

$$
T_{4}=\inf \left\{t>T_{3} ; B_{t}+\sup _{T_{3} \leq s \leq t}\left(-B_{s}\right)=\hat{W}_{T_{3}}\right\}
$$

et sur l'intervalle $\left[T_{3} ; T_{4}\right]$ on a

$$
W_{t}=B_{t}+\sup _{T_{3} \leq s \leq t}\left(-B_{s}\right) .
$$

Il est aussi clair que la suite $\left(T_{n}\right)$ croît vers l'infini. Cela découle par exemple de ce que $T_{2 n}-T_{2 n-1} \geq T_{n}^{\prime}$, où les variables

$$
T_{n}^{\prime}=\inf \left\{t>0 ; B_{T_{2 n-1}+t}+\sup _{s \leq t}\left(-B_{T_{2 n-1}+s}\right)=\frac{\varepsilon}{1-a}\right\}
$$

sont indépendantes, équidistribuées, strictement positives. 
Remarque. La démonstration ci-dessus, en particulier la construction de la suite $\left(T_{n}\right)$, utilise de manière cruciale le fait qu'on ait $W_{0}>0$. On peut aussi chercher à résoudre l'équation (6.m) lorsque $\varepsilon=0$. Pour $a<\frac{1}{2}$, un argument de point fixe montre encore qu'il y a existence et unicité d'une solution forte. La question semble plus difficile lorsque $1 / 2 \leq a<1$. Une réponse affirmative permettrait de simplifier certains des arguments de la partie 5.

\section{REFERENCES}

1. L. Dubins and G. Schwarz, On continuous martingales, Proc. Nat. Acad. Sci. U.S.A. 53 (1965), 913-916.

2. R. Durrett, $A$ new proof of Spitzer's result on the winding of two dimensional Brownian motion, Ann. Probab. 10 (1982), 244-246.

3. __ Brownian motion and martingales in analysis, Wadsworth, Monterey, Calif., 1984.

4. A. Dvoretzky and P. Erdös, Some problems on random walk in space, Proc. Second Berkeley Sympos. on Math. Stat. and Probability, Univ. of California Press, Berkeley, 1951, pp. 353367.

5. R. K. Getoor and M. J. Sharpe, Conformal martingales, Invent. Math. 16 (1972), 271-308.

6. K. Itô, Poisson point processes attached to Markov processes, Proc. Sixth Berkeley Sympos. on Math. Stat. and Probability, vol. 3, Univ. of California Press, Berkeley, 1970, pp. 225-239.

7. Th. Jeulin, Application de la théorie du grossissement à l'étude des temps locaux browniens, Grossissements de Filtrations: Exemples et Applications, Lecture Notes in Math., vol. 1118, Springer-Verlag, Berlin, 1985, pp. 197-304.

8. Th. Jeulin and M. Yor, Sur les distributions de certaines fonctionnelles du mouvement brownien, Séminaire de Probabilités XV, Lecture Notes in Math., vol. 850; Springer-Verlag, Berlin, 1981.

9. F. B. Knight, A reduction of continuous square-integrable martingales to Brownian motion, Lecture Notes in Math., vol. 190, Springer-Verlag, Berlin, 1971, pp. 19-31.

10. __ On sojourn times of killed Brownian motion, Séminaire de Probabilités XII, Lecture Notes in Math., vol. 649, Springer-Verlag, Berlin, 1978, pp. 428-445.

11. J. F. Le Gall, Sur les fonctions polaires pour le mouvement brownien, Séminaire de Probabilités XXII, Lecture Notes in Math., vol. 1321, Springer-Verlag, Berlin, 1988, pp. 186-189.

12. J. F. Le Gall and M. Yor, Etude asymptotique de certains mouvements browniens complexes avec drift, Probab. Th. Rel. Fields 71 (1986), 183-229.

13. __ Excursions browniennes et carrés de processus de Bessel, C. R. Acad. Sci. Paris Ser. I 303 (1986), 73-76.

14. __ Sur certaines lois limites intervenant dans l'étude des enlacements du mouvement brow nien dans $\mathbf{R}^{3}$ (en préparation).

15. __ Etude asymptotique des enlacements du mouvement brownien autour des droites de l'espace, Probab. Th. Rel. Fields 74 (1987), 617-635.

16. P. Messulam and M. Yor, On D. Williams' "pinching method" and some applications, J. London Math. Soc. (2) 26 (1982), 348-364.

17. J. W. Pitman and M. Yor, A decomposition of Bessel bridges, Z. Wahrsch. Verw. Gebiete 59 (1982), 425-457.

18. __ Asymptotic laws of planar Brownian motion, Ann. Probab. 14 (1986), 733-779.

19. F. Spitzer, Some theorems concerning 2-dimensional Brownian motion, Trans. Amer. Math. Soc. 87 (1958), 187-197.

20. D. Williams, A simple geometric proof of Spitzer's winding number formula for 2-dimensional Brownian motion, University College, Swansea, unpublished manuscript. 
21. _ Diffusions, Markov processes and martingales, Vol. I: Foundations, Wiley, New York, 1979.

22. M. Yor, Sur quelques approximations d'intégrales stochastiques, Séminaire de Probabilités XI, Lecture Notes in Math., vol. 581, Springer-Verlag, Berlin, 1977, pp. 518-528.

Laboratoire de Probabilites, Universite Pierre et Marie Curie, F 75252 Paris Cedex 05, FRANCE 